\title{
Measurements and Mathematical Formalism of Quantum Mechanics *
}

\author{
D.A.Slavnov \\ Department of Physics, Moscow State University, \\ Moscow 119899, Russia.E-mail: slavnov@goa.bog.msu.ru
}

\begin{abstract}
A scheme for constructing quantum mechanics is given that does not have Hilbert space and linear operators as its basic elements. Instead, a version of algebraic approach is considered. Elements of a noncommutative algebra (observables) and functionals on this algebra (elementary states) associated with results of single measurements are used as primary components of the scheme. On the one hand, it is possible to use within the scheme the formalism of the standard (Kolmogorov) probability theory, and, on the other hand, it is possible to reproduce the mathematical formalism of standard quantum mechanics, and to study the limits of its applicability. A short outline is given of the necessary material from the theory of algebras and probability theory. It is described how the mathematical scheme of the paper agrees with the theory of quantum measurements, and avoids quantum paradoxes.
\end{abstract}

\section{INTRODUCTION}

The development of quantum theory has revolutionized physics. This may be a cliche, but it is the truth. Indeed, within quantum physics, a huge number of phenomena have been described that resisted treatment within classical physics. A plethora of new technologies have been developed based on quantum physics.

However, like any revolution, the quantum revolution has its back side. Imperceptively, substitution of notions had taken place in physics. In quantum physics, "to explain a phenomenon" means "to give a mathematical description of the phenomenon."

The origin of this substitution is understandable. Modern quantum physics is an axiomatic theory based on mathematical axioms [1]. The axioms are very convenient for constructing a powerful mathematical formalism. At the same time, the axioms are almost completely detached from our intuitive notions [2]. Most theoreticians in physics support the opinion that physical intuition based on classical concepts is useless in quantum theory. Thus, theory can be constructed starting from a more or less arbitrary set of mathematical axioms. The only requirements are that the axioms should be consistent, and the consequences they imply should account for a wide enough range of experimental data. In this way, in passing, the quantum revolution substituted explanations of physical phenomena with mathematical descriptions.

Modern quantum mechanics is based on the following postulates:

I. The state of a physical system is described with a vector $|\Psi\rangle$ of a Hilbert space, or with a statistical operator (density matrix) in this space;

$I I$. Observables $\hat{D}$ of the system are described with self adjoint operators $\hat{\mathcal{D}}$;

$I I I$. The average value of observable $\hat{D}$ over the state $|\Psi\rangle$ equals the expectation value $\langle\Psi|\hat{\mathcal{D}}| \Psi\rangle$.

${ }^{*}$ Physics of Particles and Nuclei, 2007, Vol. 38, No. 2, pp. 147-176. 
Why does Hilbert space have any relation to the state of a physical system? Why does operator $\hat{\mathcal{D}}$ correspond to the observable $\hat{D}$ ? Why is the average value of the observable $\langle\Psi|\hat{\mathcal{D}}| \Psi\rangle$. All these questions are considered impertinent.

The slogan "Victors need never explain, you cannot question success" has overcome standard quantum mechanics. The huge number of excellent results obtained from the above axioms allows one to wave away the impertinent whys with a clear conscience.

Despite this, doubts remain. On the other hand, the excellent results obtained with the above axioms cannot be attributed to coincidences. May it be that statements $I-I I I$ should not be taken as primary postulates, that they would better be associated with more fundamental theses related more directly to physics?

If so, it should be possible to expose conditions for validity of statements $I-I I I$. In other words, it should be possible to establish the range of applicability of quantum mechanics. This may settle the controversies over the quantum paradoxes, which have agitated the physics community starting almost from the origin of quantum mechanics.

They break relations between quantum and classical physics. In the latter, the states and the observables are described with completely different mathematical constructs. Overall, the classicalto-quantum relation comes out rather strange. On the one hand, classical physics is considered to be a limiting case of quantum physics, i.e., it is a derivable theory. On the other hand, formulation of quantum mechanics requires the notion of interaction of a quantum object with a measuring device obeying the classical description [3]. Logically, this is a vicious circle. To break it, one states that classical logic is not valid in quantum physics, and it is to be replaced with quantum logic.

In this way, quantum theory implies, on top of other things, a revolution in logic. However, in contrast to the quantum revolution in physics, the revolution in logic has not been productive. Besides, a consistent and self contained quantum logic is yet to be created. The isolated statements available on this subject are either a reformulation of the postulates $I-I I I$, or their corollary. Consequently, in physical practice, statements of quantum logic are not used, and the underlying postulates $I-I I I$ are used directly.

There is another annoyance in the standard formulation of quantum mechanics. In its basics, quantum mechanics is a statistical theory. Therefore, it should be based on probability theory. Presently, probability theory (in the Kolmogorov formulation [4])is a rather solidified mathematical subject. However, it is assumed that this probability theory is not fit for quantum mechanics, and a dedicated quantum probability theory is required. Thus, on top of the above, quantum theory requires a revolution in mathematics. As with quantum logic, no success has been achieved in this direction. At best, detached statements are available for the new probability theory, and, in fact, they are again corollaries of the postulates $I-I I I$ (e.g., see [5]).

We conclude that there is a gap between quantum theory and mathematics; the latter stays within classical logic and probability theory. The above implies that it is highly desirable to design a mathematical scheme that would be equally fit for both classical and quantum physics. It would be nice, if the rules of the game, or, in philosophers' parlance, the paradigm of the scheme were classical. By classical paradigm, we mean here one that respects classical formal logic and incorporates the notion of causative relations between physical phenomena, as well as between logical statements. Next, it should imply the existence of physical realities that carry causes of physical phenomena. Additionally, it would conjecture that probability propositions obey classical Kolmogorov probability theory.

Usually, it is assumed that the above statements are incompatible with the mathematical scheme accepted in quantum mechanics. Here, we attempt to prove the opposite. Our exposition is not based on postulates $I-I I I$. Instead, we use the algebraic approach [6, 7, 8]. We formulate axioms within this approach that are, firstly, more fundamental than the postulates $I-I I I$, and, secondly, are much more amenable to intuitive understanding [9, 10, 11.

Admittedly, a psychological barrier must be crossed at this point. The formalism of Hilbert 
space has become standard in quantum mechanics. Due to this, it is perceived as intuitively understandable. Physical intuition is replaced here with a particular mathematical formalism. In contrast, the formalism of algebraic theory is not so familiar to most physicists. Due to this, statements employing the language developed in the theory of algebras are perceived as more involved than the parallel statements employing the language of Hilbert space. This is despite the fact that the algebraic statements are more elementary as a rule. We help to step over this psychological barrier in the next section with an outline of elementary facts taken from the theory of algebras.

\section{ELEMENTS OF THE THEORY OF ALGEBRAS}

We outsource definitions and statements from [6, 12, 13, 14, 15].

Definition 1. A set $\mathfrak{L}$ is called a complex (real) linear space if

(a) for any complex (real) number $\alpha$ and any element $\hat{U} \in \mathfrak{L}$, there is a unique element $\alpha \hat{U} \in \mathfrak{L}$;

(b) for any two elements $\hat{U}, \hat{V} \in \mathfrak{L}$, there is a unique element $\hat{U}+\hat{V} \in \mathfrak{L}$;

(c) operations (a) and (b) have the familiar properties of multiplication and addition, respectively.

Definition 2. A complex (real) linear space $\mathfrak{L}$ is called a complex (real) algebra $\mathfrak{A}$ if a multiplication operation is defined for any elements $\hat{U}, \hat{V}, \hat{W} \in \mathfrak{A}$ that satisfy the following properties:

(a) $\hat{U} \hat{V} \in \mathfrak{A}$;

(b) $(\hat{U}+\hat{V}) \hat{W}=\hat{U} \hat{W}+\hat{V} \hat{W}, \hat{U}(\hat{V}+\hat{W})=\hat{U} \hat{V}+\hat{U} \hat{W}$;

(c) $\alpha(\hat{U} \hat{V})=(\alpha \hat{U}) \hat{V}=\hat{U}(\alpha \hat{V})$.

Definition 3. Algebra $\mathfrak{A}$ is called an associative algebra if the relation $\hat{U}(\hat{V} \hat{W})=(\hat{U} \hat{V}) \hat{W}$ holds for any elements $\hat{U}, \hat{V}, \hat{W} \in \mathfrak{A}$.

Definition 4. Algebra $\mathfrak{A}$ is called a commutative algebra if the relation $\hat{U} \hat{V}=\hat{V} \hat{U}$ holds for any elements $\hat{U}, \hat{V} \in \mathfrak{A}$.

EXAMPLES:

(a) the set of all real continuous bounded functions of one variable is a real algebra;

(b) the set of all complex continuous bounded functions is a complex algebra;

(c) the set of bounded linear operators of a Hilbert space is a complex algebra;

(d) the set of mutually commuting bounded Hermitian linear operators of a Hilbert space is a real algebra;

(e) the set of all bounded Hermitian linear operators of a Hilbert space is not an algebra.

Definition 5. A mapping $\hat{U} \rightarrow \hat{U}^{*}$ of a complex algebra $\mathfrak{A}$ onto itself (i.e., $\left(\hat{U}, \hat{U}^{*} \in \mathfrak{A}\right)$ ) is called an involution if the following holds for any complex number $\alpha$ and any $\hat{U}, \hat{V} \in \mathfrak{A}$ :

(a) $(\hat{U}+\hat{V})^{*}=\hat{U}^{*}+\hat{V}^{*}$,

(b) $(\alpha \hat{U})^{*}=\alpha^{*} \hat{U}^{*}$,

(c) $(\hat{U} \hat{V})^{*}=\hat{V}^{*} \hat{U}^{*}$

(d) $\hat{U}^{* *}=\hat{U}$.

EXAMPLES:

(a) a complex conjugation is an involution for $\mathfrak{A}$ that is the set of all complex bounded functions of a single variable;

(b) a Hermitian conjugation is an involution for $\mathfrak{A}$ that is the set of all bounded linear operators of a Hilbert space. 
Definition 6. A complex algebra equipped with an involution operation is called an involutive algebra.

REMARK. The identity transformation is an involution for any real commutative algebra.

Definition 7. If $\hat{U}^{*}=\hat{U}(\hat{U} \in \mathfrak{A})$, the element $\hat{U}$ is called Hermitian.

Definition 8. An element $\hat{I} \in \mathfrak{A}$ satisfying the relations $\hat{I} \hat{U}=\hat{U} \hat{I}=\hat{U}$ for any $\hat{U} \in \mathfrak{A}$ is called the unit element of the algebra.

Proposition 1. Any algebra either has a unit element or can be supplemented with an element satisfying the properties of the unit element.

In the following, we consider the algebras with unity.

Definition 9. Element $\hat{U}^{-1} \in \mathfrak{A}$ is called the inverse of $\hat{U}$, if $\hat{U}^{-1} \hat{U}=\hat{U} \hat{U}^{-1}=\hat{I}$.

Definition 10. The spectrum $\sigma(\hat{U} ; \mathfrak{A})$ of the element $\hat{U}$ in the algebra $\mathfrak{A}(\hat{U} \in \mathfrak{A})$ is the set of all numbers $\lambda$ for which the element $\lambda \hat{I}-\hat{U}$ has no inverse element within the algebra $\mathfrak{A}$.

Definition 11. The number $r=\sup \{|\lambda| ; \quad \lambda \in \sigma(\hat{U} ; \mathfrak{A})\}$ is called the spectral radius of the element $\hat{U}$.

Definition 12. A subset $\mathfrak{Q}$ of an algebra $\mathfrak{A}$ is called a subalgebra if $\mathfrak{Q}$ is an algebra for the available definition of multiplication and addition.

Definition 13. Let $\mathfrak{Q}$ be a real commutative subalgebra of algebra $\mathfrak{A}$. The subalgebra $\mathfrak{Q}$ is called a maximal commutative real subalgebra if it is not contained in any other such subalgebra of $\mathfrak{A}$.

Generally, the spectrum $\sigma(\hat{U} ; \mathfrak{Q})$ of element $\hat{U}$ with respect to algebra $\mathfrak{Q}$ may not coincide with the spectr $\sigma(\hat{U} ; \mathfrak{A})$ of the same element with respect to algebra $\mathfrak{A}$. However, the following proposition holds.

Proposition 2. If $\mathfrak{Q}$ is a maximal real commutative subalgebra of algebra $\mathfrak{A}$ and $\hat{U} \in \mathfrak{Q}$, then $\sigma(\hat{U} ; \mathfrak{Q})=\sigma(\hat{U} ; \mathfrak{A})$.

Definition 14. A set $\mathfrak{J}_{l}$ of elements belonging to algebra $\mathfrak{A}$ is called its left ideal if:

(a) $\mathfrak{J}_{l} \neq \mathfrak{A}$,

(b) $\mathfrak{J}_{l}$ is a linear subspace of $\mathfrak{A}$,

(c) relations $\hat{U} \in \mathfrak{J}_{l}, \hat{V} \in \mathfrak{A}$ imply $\hat{V} \hat{U} \in \mathfrak{J}_{l}$.

Right ideals are defined similarly. A set $\mathfrak{J}$ of elements from algebra $\mathfrak{A}$ that is simultaneously a left and right ideal is called two-sided ideal.

Definition 15. Let $\mathfrak{J}$ be a two-sided ideal of algebra $\mathfrak{A}$. Elements $\hat{U}$ and $\hat{V}$ are called equivalent with respect to $\mathfrak{J}$ if $\hat{U}-\hat{V} \in \mathfrak{J}$. A set of all elements equivalent to each other is called a residue class of algebra $\mathfrak{A}$. 
Definition 16. The set of all residue classes of algebra $\mathfrak{A}$ is called a factor algebra, and denoted $\mathfrak{A} / \mathfrak{J}$.

Proposition 3. The set $\mathfrak{A} / \mathfrak{J}$ equipped with operations of class multiplication by numbers and class additions introduced as respective operations for representatives of the classes becomes an algebra. In other words, a factor-algebra is an algebra.

Definition 17. An involutive algebra is called normed if a norm $\|\hat{U}\|$ is defined for each element $\hat{U}$. The norm is a nonnegative number satisfying the following conditions:

(a) $\|\alpha \hat{U}\|=|\alpha|\|\hat{U}\|$

(b) $\|\hat{U}+\hat{V}\| \leq\|\hat{U}\|+\|\hat{V}\|$;

(c) $\left\|\hat{U}^{*}\right\|=\|\hat{U}\|$

(d) $\|\hat{U} \hat{V}\| \leq\|\hat{U}\|\|\hat{V}\|$

(e) $\|\hat{U}\|=0$ implies $\hat{U}=0$.

Definition 18. A quantity $\|\hat{U}\|$ satisfying all the above conditions apart of condition (e) is called a semi norm.

Definition 19. A sequence of elements of a normed spac $\left\{\hat{U}_{n}\right\}$ is called fundamental if for any $\varepsilon>0$ there exists a number $N(\varepsilon)$, such that the inequalities $n>N(\varepsilon)$ and $m>N(\varepsilon)$ imply the inequality $\left\|\hat{U}_{n}-\hat{U}_{m}\right\|<\varepsilon$.

Definition 20. A normed space in which any fundamental sequence is convergent in norm to an element of this space is called complete.

Definition 21. A complete normed space is called a Banach space.

Proposition 4. Any normed space can be completed to a Banach space.

Definition 22. An involutive associative algebra that is a Banach space (a Banach algebra) with the norm satisfying the additional requirement $\left\|\hat{U}^{*} \hat{U}\right\|=\|\hat{U}\|^{2}$ is called a $\mathrm{C}^{*}$-algebra.

DeFinition 23. A mapping $\hat{U} \rightarrow \hat{U}^{\prime}$ of an involutive algebra $\mathfrak{A}(\hat{U} \in \mathfrak{A})$ into involutive algebra $\mathfrak{A}^{\prime}\left(\hat{U}^{\prime} \in \mathfrak{A}^{\prime}\right)$ is called a homomorphism from algebra $\mathfrak{A}$ to algebra $\mathfrak{A}^{\prime}$ if $\hat{U} \rightarrow \hat{U}^{\prime}$ and $\hat{V} \rightarrow \hat{V}^{\prime}$ implies the following relations: $\hat{U}^{*} \rightarrow \hat{U}^{\prime *}, \alpha \hat{U} \rightarrow \alpha \hat{U}^{\prime}, \hat{U}+\hat{V} \rightarrow \hat{U}^{\prime}+\hat{V}^{\prime}$, and $\hat{U} \hat{V} \rightarrow \hat{U}^{\prime} \hat{V}^{\prime}$.

A homomorphism may map several elements of $\mathfrak{A}$ into a single element of $\mathfrak{A}^{\prime}$.

Definition 24. If a homomorphism is a one-to-one mapping, it is called an isomorphism.

Definition 25. An isomorphic mapping of an algebra onto itself is called an automorphism.

Definition 26. A homomorphism of a commutative associative real (complex) algebra $\mathfrak{A}$ into the set of real (complex) numbers is called a character of the algebra.

Definition 27. A homomorphism of algebra $\mathfrak{A}$ into a set of linear operators of a Hilbert space $\mathfrak{H}$ is called a representation of the algebra.

Definition 28. A mapping from normed algebra $\mathfrak{A}$ to normed algebra $\mathfrak{A}^{\prime}$ is called isometric if $\hat{U} \rightarrow \hat{U}^{\prime}$ implies $\|\hat{U}\| \rightarrow\left\|\hat{U}^{\prime}\right\|$. 
Definition 29. Mapping $\hat{U} \rightarrow \varphi(\hat{U})$ from algebra $\mathfrak{A}$ into complex numbers is called a linear functional if $\varphi(\alpha \hat{U})=\alpha \varphi(\hat{U})$ and $\varphi(\hat{U}+\hat{V})=\varphi(\hat{U})+\varphi(\hat{V})$. Here, $\hat{U}, \hat{V} \in \mathfrak{A}$ and $\alpha, \varphi(\hat{U})$ are complex numbers.

DeFinition 30. Linear functional $\varphi$ on an involutive algebra $\mathfrak{A}$ is called positive if $\varphi\left(\hat{U} \hat{U}^{*}\right) \geq 0$ for any $\hat{U} \in \mathfrak{A}$.

Proposition 5. If $\varphi(\hat{U})$ is a positive functional, then (a) $\varphi\left(\hat{U}^{*}\right)=\varphi^{*}(\hat{U})$

(b) $\left|\varphi\left(\hat{U}^{*} \hat{V}\right)\right|^{2} \leq \varphi\left(\hat{U}^{*} \hat{U}\right) \varphi\left(\hat{V}^{*} \hat{V}\right)$.

Proposition 6. A positive functional on a Banach algebra is continuous.

Proposition 7. If $\varphi(\hat{U})(\hat{U} \in \mathfrak{A})$ is a character of an associative commutative algebra $\mathfrak{A}$, then (a) $\varphi(0)=0$

(b) $\varphi(\hat{I})=1$,

(c) $\varphi\left(\hat{U} \hat{U}^{*}\right) \geq 0$.

Thus, character is a positive functional on algebra $\mathfrak{A}$.

Proposition 8. For a Banach algebra $\mathfrak{A}$, the following holds for the set $\{\varphi(\hat{U})\}$ of all its characters:

(a) $\lambda=\varphi(\hat{U}) \in \sigma(\hat{U} ; \mathfrak{A})$;

(b) if $\lambda \in \sigma(\hat{U} ; \mathfrak{A})$, there exists $\varphi(\hat{U}) \in\{\varphi(\hat{U})\}$ for which $\lambda=\varphi(\hat{U})$.

Definition 31. Element $\hat{p}$ of algebra $\mathfrak{A}$ is called a projector if $\hat{p}^{*}=\hat{p}, \hat{p}^{2}=\hat{p}$.

Definition 32. Projector $\hat{p}_{\lambda} \neq 0$ is called minimal if relations $\hat{p}_{\lambda} \hat{p}_{\mu}=\hat{p}_{\mu} \hat{p}_{\lambda}=\hat{p}_{\mu}$ imply either $\hat{p}_{\mu}=0$ or $\hat{p}_{\mu}=\hat{p}_{\lambda}$.

Proposition 9. If $\mathfrak{A}$ is an algebra of bounded linear operators in a Hilbert space $\mathfrak{H}$, any minimal projector is a projector onto a one-dimensional subspace of space $\mathfrak{H}$.

Definition 33. Sequence $\left\{\hat{U}_{n}\right\}$ of elements of algebra $\mathfrak{A}$ is called convergent in weak topology to an element $\hat{U}$ if the relation $\varphi\left(\hat{U}_{n}\right) \rightarrow \varphi(\hat{U})$ holds for any linear bounded positive functional $\varphi$.

Definition 34. A set $G$ of elements belonging to Banach algebra $\mathfrak{A}$ is called a generating set of the algebra if the smallest closed subalgebra containing $G$ coincides with $\mathfrak{A}$.

Definition 35. The Boolean algebra of a set $\Omega$ is the totality of all subsets of $\Omega$ supplied with the following operations:

(a) operation of logical addition - the union of subsets;

(b) operation of logical multiplication - the intersection of subsets;

(c) operation of logical negation - the complement of subsets to the set $\Omega$.

Definition 36. A Boolean algebra is called complete with respect to an algebraic operation if the operation results in an element of the original algebra.

Definition 37. A Boolean algebra is called $\sigma$-algebra under the following conditions:

(a) it contains the set $\Omega$ and the empty set $\emptyset$; 
(b) it contains the complement to $\Omega$ of any subset belonging to the algebra;

(c) it is closed under countable number of unions and intersections of subsets.

Definition 38. A set $\Omega$ with a specific $\sigma$-algebra selected is called a measurable set.

Hereafter, we refer the above definitions and propositions in the following style: (D.35.b) means definition 35 point b, (P.7.c) means proposition 7, point .

\section{OBSERVABLES, MEASUREMENTS, AND STATES}

Let us turn to physics. We aim at formulating the basic postulates of quantum mechanics. We will try to use as postulates statements admitting direct experimental checks, in contrast to checks of distant consequences. Otherwise, we risk making redundant assumptions and running into contradictions. Correspondingly, we start with physical phenomena, and tune the mathematical formalism, instead of looking for a physical interpretation of a preset mathematical scheme.

The basic notion in studying physical systems is the notion of the observable. It seems to be a self evident notion not requiring an exact definition. Heuristically, an observable is an attribute of a physical system that can be supplied with a number value via a measurement procedure.

REMARK. In what follows, we assume that units are fixed, and all the observables can be considered as dimensionless.

Both classical and quantum physics have observables as their basic notions. Despite this, in mathematical formalisms of classical and quantum physics, the observables correspond to different mathematical objects. Let us attempt unification. Aiming at this, we try to isolate the relevant mathematical features of observables, separating them form the ones that are customarily attributed to observables for purposes of facilitating development of the mathematical formalism.

REMARK. Not infrequently, a number of observables are not subject to changes for a system under study. For example, in the study of interactions between photons and electrons, the masses of electrons and photons and the charge of an electron can be given beforehand. It is convenient to exclude such quantities from the set of observables, considering them as parameters involved in the definition of the system.

Under a measurement, the system is influenced by the measuring device. Depending on the kind of this influence, measurements can be classified into two kinds: reproducible and irreproducible. Reproducible measurements are characterized by the property that, despite the perturbation introduced upon each measurement, repeated measurement of the same observable with the same or some other device yields the same result. It is assumed that between the measurements, the system is not affected by external influences, and we are able to take into account the changes in the values of the observables due to free evolution.

The problem of reproducibility is of particular interest in the case when a number of observables are measured for the same physical system. Let us first measure observabl $\hat{A}$, then observable $\hat{B}$, then again observable $\hat{A}$ (probably, with a different device), and, finally, observable $\hat{B}$. If the results of the repeated measurements coincide with the original ones, we call such measurements compatible. If there are devices allowing one to make combined measurements of observables $\hat{A}$ and $\hat{B}$, we call such observables compatible or simultaneously measurable.

Experiments demonstrate that all the observables are compatible for classical systems. In contrast to this case, there are both compatible and incompatible observables in the quantum case. 
In the standard quantum mechanics, this fact is incorporated into the complementarity principle [16]. We simply consider it as a manifestation of the necessity of incompatible devices for measuring two incompatible observables [17].

We denote the set of all observables by $\mathfrak{A}_{+}$, and its maximal subset of compatible observables by $\mathfrak{Q}_{\xi}$. The subscript $\xi$ distinguishes between different maximal subsets of incompatible observables. The subscript $\xi$ takes values in the set $\Xi$. Evidently, $\Xi$ consists of a single element for a classical system. For a quantum system, this set contains more than one element. Subsequently, we will see that this set is infinite, and even has the cardinality of continuum. One and the same observable may simultaneously belong to different subsets $\mathfrak{Q}_{\xi}$.

Experiments demonstrate that for any two compatible observables $\hat{A}$ and $\hat{B}$, there exists a third observable $\hat{D}$ possessing the following properties. First, it is compatible both with $\hat{A}$ and $\hat{B}$. Second, the outcomes of simultaneous measurements of the observables $\hat{A}, \hat{B}$, and $\hat{D}$ (for one and the same physical system) satisfy the relation

$$
A+B=D .
$$

In fact, simultaneity is not particularly relevant. Compatibility of these observables suffices. Despite this, for brevity, we characterize such a situation saying that the observables are measured simultaneously.

Relation (1) holds regardless of the particular outcomes of the measurements. In view of this, it is possible to assume that the observables themselves are related by a similar relation:

$$
\hat{A}+\hat{B}=\hat{D} \text {. }
$$

In this way, it is possible to equip a set $\mathfrak{Q}_{\xi}$ with addition. Analogously, multiplication of elements, and multiplication by real numbers are introduced. Experiments show that each of the subsets $\mathfrak{Q}_{\xi}$ has the properties of real associative commutative algebra. Thus, the mathematical description of observables maintains that they are elements of some algebra. Until now, we have justified this statement only for compatible observables. We will see that it can be extended to incompatible observables as well.

Relating to each physical observable (via compatible measurements) the result of a measurement,

$$
\hat{A} \rightarrow A=\varphi_{\xi}(\hat{A}),
$$

we define a functional on the algebra $\mathfrak{Q}_{\xi}$. By the definition of algebraic operations in $\mathfrak{Q}_{\xi}$, this functional is one of the characters of algebra $\mathfrak{Q}_{\xi}$ (see (D.26)).

Any real measurement of an observable gives it a finite value. Reversing this fact, physical observables (i.e., the ones measured in real experiments) are only the observables $\hat{A}$ satisfying the relation

$$
\sup _{\xi} \sup _{\varphi_{\xi}}\left|\varphi_{\xi}(\hat{A})\right|<\infty .
$$

In the following, we will see that the boundedness of functionals $\varphi_{\xi}(\cdot)$ is not an insurmountable obstacle for considering within the theory unbounded observables. Such observables are commonplace in standard quantum mechanics.

We summarize the above considerations in the following postulates

Postulate 1. A set $\mathfrak{Q}_{\xi}$ of compatible observables can be equipped with the structure of a real associative commutative algebra. Conversely, if observables belong to one and the same real associative commutative algebra, they are compatible.

Postulate 2. For a classical system, all observables are compatible. 
Postulate 3. The outcomes of simultaneous measurements of observables belonging to algebra $\mathfrak{Q}_{\xi}$ are described by real bounded (in the meaning of inequality (2) functional $\varphi_{\xi}(\cdot)$, which is a character of algebra $\mathfrak{Q}_{\xi}$.

Above, we repeatedly used the notion of physical system. It is intuitively clear what this notion means. In view of this, we will not try to give it a physical interpretation. However, constructing mathematical formalism requires clear understanding of the meaning of the expression "a given physical system."

In the following, we assume that a physical system is given if a number of conditions are met. First, a set of system observables $\mathfrak{A}_{+}$is given. Quantities whose values are known beforehand and are not varied are not included in $\mathfrak{A}_{+}$Instead, their values are considered given. Second, sets of compatible observables $\mathfrak{Q}_{\xi}(\xi \in \Xi)$. are given. These sets are subsets of set $\mathfrak{A}_{+}$. Third, relations between observables are given. Relations between compatible observables are defined by their membership in $\mathfrak{Q}_{\xi}$. Relations between incompatible observables will be considered later. Fourth, the dynamics of the system is given. Since we aim at unified description of classical and quantum systems, we will not use a concrete method of describing system dynamics. We simply take that observables can depend on time, and the statement "relations between observables are given" implies also that relations between observables at consecutive moments of time are given.

REMARK. If the physical system under consideration is a conservative one, because of uniformity of time, it is appropriate to take that dynamics is described with a time-dependent automorphism on the algebra of observables. However, if the system endures external perturbations depending on time, description of dynamics may be more involved. In particular, it may require modifications of the algebra of observables itself.

Apart from consideration of the whole Universe as a given physical system, any physical system is a subsystem of a larger system. Mathematically, this means that the observables of the system form a subset of observables of another system. Selection of the subset can be carried out by various features. The first among these is localization.

Any domain $\mathcal{O}$ of the four-dimensional space-time is related to a set of observables whose values can be obtained via measurements carried out within the domain $\mathcal{O}$. Such observables are called local (localized in the domain $\mathcal{O}$ ) [6, 7]. Strictly speaking, all observables should be considered as local. However, global (quasi-local) observables are normally also considered in the theory. They are constructed as limits of sequences of local observables.

Using the localization feature, a physical system is mathematically a set of observables localized in a domain. However, other features should also be considered in practice. For example, consider a solid body. Generally, a solid body is characterized by a huge number of observables. Some of them characterize the solid body as a whole. These observables can be considered within the classical physics. Other observables characterize individual molecules. These observables cannot be described within the classical physics. At the same time, both types of observables are localized in the domain $\mathcal{O}$, occupied with the solid body. Thus, in this case the selecting feature of the system is not only the localization of the observables, but also their classical character.

This example demonstrates also that it may be possible to single out from a quantum system characterized by a multitude of compatible and incompatible observables a classical subsystem characterized only with compatible observables. In this, isolation of subsystem from the other part of the system is not assumed. For example, elastic collisions are successfully described within classical physics. In these processes, molecules described by quantum physics are actively involved. Within the classical description, participation of molecules can be taken into account via boundary conditions, or via effective external field. 
Let us discuss now the notion of the state of a physical system. We start with discussion of classical systems. In this case, the state is the attribute of the system that defines the outcomes of all measurements of the observables unambiguously. From Newton's times, the locality principle has been accepted in physics. It assumes, in particular, that the state of a localized physical system is determined by the internal characteristics of the system and the characteristics of the external fields acting on the system that refer to the localization domain of the physical system. By the classical paradigm, there exists a local reality governing the state of the system.

Mathematically, a state is given by a point in a phase space. It is assumed here that dynamics of the system is set. In the approach of this paper, this way of setting the state is inconvenient. First, it is hard to carry it over to the quantum case. Second, it is rigidly associated with a particular way of setting the dynamics. In particular, it assumes the introduction of canonically conjugate variables. However, it is easily seen that this way of setting a state is merely a particular way of setting a real functional on the algebra of observables. This functional is a character of the algebra. If we do not restrict our consideration to a particular variant, a state of a classical system can be defined as a character of the algebra of observables of the system.

Let us now turn to the quantum case. The set $\mathfrak{A}_{+}$of quantum observables cannot be equipped with the structure of associative commutative algebra. In view of this, it is not possible to directly carry over the classical definition of the state to the quantum case. However, it is possible to consider the set $\mathfrak{A}_{+}$as the totality of subsets $\mathfrak{Q}_{\xi}(\xi \in \Xi)$. And each subset $\mathfrak{Q}_{\xi}$ can be considered as a set of observables corresponding to a classical subsystem of the quantum system. Certainly, such a classical subsystem is not isolated form the rest of the system. However, the possibility of selecting a subsystem is not strictly stipulated by isolation from the rest of the system.

The state of each such subsystem is mathematically defined as before via a functional $\varphi_{\xi}(\cdot)$ defined on the algebra $\mathfrak{Q}_{\xi}$, which is a character of this algebra. In this context we introduce a new notion - the notion of elementary state.

Definition 39. The elementary state of a physical system is a set $\varphi=\left[\varphi_{\xi}\right](\xi \in \Xi)$ of functionals $\varphi_{\xi}$. Each of them is a character of the corresponding algebra $\mathfrak{Q}_{\xi}$. The sets $\mathfrak{Q}_{\xi}$ are the maximal subsets of the set $\mathfrak{A}_{+}$having the structure of real associative commutative algebra.

The term "state" is justified also in the quantum case. Indeed, in any particular measurement and even in a set of compatible measurements, we can at best find values for a set of compatible observables. All these observables belong to a single algebra $\mathfrak{Q}_{\xi}$. Therefore, their values are determined by the corresponding functional $\varphi_{\xi}$. All such functionals are defined by elementary state $\varphi$. Consequently, it fixes the results of all possible measurements. In the standard quantum mechanics, another mathematical object is associated with a state. In view of this, we call $\varphi=\left[\varphi_{\xi}\right]$ an "elementary state." This term is not used in the standard quantum mechanics. This stage of considerations is concluded with the following postulate.

Postulate 4. The outcome of any particular experiment measuring the observables of a physical system is determined by the elementary state of the system.

The last postulate gives nothing new in the classical case. On the contrary, it is a completely unconventional postulate in the quantum case. Moreover, there are multiple proofs that nothing like it can be true. This postulate is central to our approach. It is possible to take that an elementary state realizes the concept of "potential possibilities." This notion was introduced by Fock [18, but he did not give it a mathematical formulation.

Let us stress that we do not make extra assumptions on the properties of the functionals $\varphi_{\xi}$. In particular, we do not assume that

$$
\varphi_{\xi}(\hat{A})=\varphi_{\xi^{\prime}}(\hat{A}), \text { if } \hat{A} \in \mathfrak{Q}_{\xi} \cap \mathfrak{Q}_{\xi^{\prime}} .
$$


Certainly, equality (3) may hold for some elementary states $\varphi$. We say that an elementary state $\varphi$ is stable on the observable $\hat{A}$ if (13) holds for any $\mathfrak{Q}_{\xi}$ and $\mathfrak{Q}_{\xi^{\prime}}$ containing observable $\hat{A}$.

On the other hand, it seems to be very natural to require fulfillment of equality (3). In view of this, we have to comment on the violation of this equality.

The experimental values of observables appear as responses of a measuring device to the influences from the system under measurement. Generally, responses of different devices to one and the same influence may be different. From the standpoint of the experimenter, such data is bad. The experimenter strives for unification of the response (readings) of the devices. The unification is achieved via device calibration.

Schematically, calibration goes as follows. A measuring device serves as a reference one if it performs a reproducible measurement of an observable $\hat{A}$. With this device, observabl $\hat{A}$ is measured for a test physical system. It results in a value $A$. By the definition of a reproducible measurement, repeated measurement of the same observable with a device under calibration should yield the same value. Only a device able to pass such a test many times can be called a measuring device. Calibration is to exclude dependence of the measurement outcome on the uncontrollable influence of the device, in particular, on the uncontrollable state of the device.

However, calibration is unable to exclude dependence on a particular parameter whose value may be a property of the device. This parameter is $\xi \in \Xi$. Let us clarify the relation between parameter $\xi$ and the measuring device. Any device is dedicated depending on its construction (tuning) either to the measurement of a single observable $\hat{A}$, or to the simultaneous measurement of a set of observables. This observable (set of observables) belongs to an algebra $\mathfrak{Q}_{\xi}$. We take that a device is of type $\xi$ if it is dedicated to measurements of an observable (observables) belonging to the subset $\mathfrak{Q}_{\xi}$, and, secondly, if the outcome of the measurement of observable $\hat{A} \in \mathfrak{Q}_{\xi}$ (or set of compatible measurements) is $A_{\xi}=\varphi_{\xi}(\hat{A})$ (set of corresponding results).

Calibration cannot ascertain if the outcome of measurement depends on parameter $\xi$ or not. Indeed, calibration starts with a reproducible measurement. After this measurement, the state of the system testing the device becomes stable on observab $\hat{A}$. Therefore, the outcome of a subsequent measurement of this observable will not in any case depend on parameter $\xi$. Any other way of checking equality (3) implies that we should put one and the same testing system under two measurements: once under measurement by a device of type $\xi$, and again by a device of type $\xi^{\prime}\left(\xi \neq \xi^{\prime}\right)$. The devices are different, and, for this reason, the measurements cannot be performed simultaneously.

Assume that the first measurement with the device of type $\xi$ has outcome $A_{\xi}=\varphi_{\xi}(\hat{A})$. If this is an irreproducible measurement, the elementary state $\varphi$ of the system under measurement will change uncontrollably. In this case, the outcome of the second measurement (with device of type $\left.\xi^{\prime}\right)$ will not be related in any way to the result of the first measurement. If the first measurement is reproducible, elementary state $\varphi$ will be replaced with $\varphi^{\prime}$ after the measurement. Since after a reproducible measurement, the elementary state becomes stable on the corresponding observable, the state $\varphi^{\prime}$ should satisfy relation $\varphi_{\xi^{\prime}}^{\prime}(\hat{A})=\varphi_{\xi}(\hat{A})$ regardless of the fulfillment of equality (3). We conclude that checking (3) is beyond our reach in any case.

Certainly, the above considerations do not guarantee that the outcomes of measurements do depend on $\xi$. They only demonstrate that such a dependence is possible. Therefore, any conclusions based on assuming the validity of equality (3), have no argumentativeness. We stress that type classification of measuring devices by values of $\xi$ is a classification by the character of interaction between the device and the system under measurement. Therefore, not only the properties of the device, but also the system under measurement (the set $\mathfrak{A}_{+}$and algebras $\mathfrak{Q}_{\xi}$ ) determine it.

The dependence of measurement outcome on the device type can be considered as a realization and concrete definition of Bohr's views [19] on the dependence of measurement outcome on the general context of experiment. At the same time, the particular variant of the dependence we suggest here does not contradict the causality principle or the conception of local reality existence. Local 
reality does not imply a definite value of any observable of the physical system under consideration. It is rather a cause effecting a particular reaction in a certain type of measuring device.

Any measuring device is a classical system, and any external influence on it can be considered as an effect of some external field acting on the device. Here, the system under measurement should be the source of the field. The device as a classical system is insensitive to microscopic quantum details of the field, only its classical characteristics matter. Therefore, in this context, this field can be considered as an effective classical field having characteristics governed by the elementary state of the system under measurement. Namely, this effective field should be considered as local reality dictating the outcome of any particular measurement.

Only when the elementary state is stable on an observable is it possible to speak of a definite value of this observable. In the classical case, set $\Xi$ contains a single element. Thus, all measuring devices belong to one and the same type. Correspondingly, all elementary states are stable on any observable, i.e., any observable has a definite value.

Measurement with a classical device cannot fix the elementary state of a quantum system unambiguously. Indeed, as the devices dedicated to measurements of incompatible observables are incompatible, only observables belonging to a single algebra $\mathfrak{Q}_{\xi}$. are accessible to a measurement in a particular experiment. As a result, only the values of the functional $\varphi_{\xi}$. will be established. The rest of the elementary state $\varphi$ will remain undetermined. Repeated measurement employing a device of another type will yield additional information, but will perturb uncontrollably the elementary state established after the first measurement. And the information obtained in the first measurement will become useless.

In view of this, we take the following definition.

Definition 40. Elementary states $\varphi$ are called $\varphi_{\xi}$-equivalent if their restrictions $\varphi_{\xi}$ on the algebra $\mathfrak{Q}_{\xi}$ are identical.

Thus, quantum measurement is only able to establish the equivalence class of the physical state under measurement. The procedure of preparation of a pure state in the standard quantum mechanics can be easily recognized in the reproducible measurement of observables belonging to algebra $\mathfrak{Q}_{\xi}$. In the following, we call this the quantum state. The definition for it reads as follows.

Definition 41. Quantum state $\Psi_{\varphi \xi}$ is the class $\{\varphi\}_{\varphi \xi} \quad \varphi_{\xi}$-equivalent elementary states that are stable on algebra $\mathfrak{Q}_{\xi}$.

In fact, this definition is convenient only for systems without identical particles. The problem is that the measuring device is unable to determine which of the identical particles has been detected. In view of this, a generalization of the definition is convenient. We will say that elementary state $\varphi$ is weakly $\varphi_{\xi}$-equivalent to elementary state $\varphi^{\prime}$ if restriction $\varphi_{\xi}$ of the elementary state $\varphi$ onto algebra

$\mathfrak{Q}_{\xi}$ coincides with restriction $\varphi_{\xi^{\prime}}^{\prime}$ of the elementary state $\varphi^{\prime}$ on the algebra $\mathfrak{Q}_{\xi^{\prime}}$ that results from $\mathfrak{Q}_{\xi}$ after replacement of the observables of one of the identical particles with the corresponding observables of another identical particle. For systems with identical particles, the equivalence involved in the definition of the quantum state should be replaced with a weak equivalence. We assume hereafter that such replacement is performed if necessary.

In this way, our quantum state characterizes not a single state but an ensemble of such objects. We share this view with Blokhintsev [20, 21, 22, 23].

\section{PROBABILITY THEORY AND QUANTUM ENSEMBLE}

Most predictions of quantum theory are probabilistic. Therefore, quantum theory should be based on probability theory. Presently, Kolmogorov probability theory is the most developed mathemat- 
ically [4]. It is commonly believed that quantum systems require dedicated quantum probability theory. Here, we advocate the view that classical Kolmogorov probability theory is quite adequate to the quantum case if one takes into account the peculiarities of quantum measurements [24].

The basic notion of Kolmogorov probability theory (see, e.g., [4, 25]) is the so-called probability space $(\Omega, \mathcal{F}, P)$.

The first component $\Omega$ is a set (space) of elementary events. The physical meaning of elementary events is not specified, but it is assumed that the events are mutually exclusive, and one and only one event is realized in each trial. In our case an elementary event appears as an elementary state $\varphi$.

REMARK. Evidently, it is not possible to use a quantum state as an elementary event, because two nonorthogonal events are not mutually exclusive. Therefore, it is indeed not possible to use Kolmogorov probability theory within the standard quantum mechanics. The classical formal logic does not fit in the standard quantum mechanical framework for similar reasons.

Apart form the elementary event, the notion of a random event, or, for brevity, event (without any adjective) is introduced. Any event $F$ is identified with a subset of the set $\Omega$. Event $F$ is assumed to be realized if any of the elementary events belonging to this subset $(\varphi \in F)$ has realized itself. It is assumed that we can establish whether an event is realized or not for any trial. There is no need to assume the same about elementary events.

Sets of subsets of the set $\Omega$ (including the set $\Omega$ itself and the empty set $\emptyset$ ) are equipped with the structure of Boolean algebra. Correspondingly, the second component of probability space is a Boolean $\sigma$-algebra $\mathcal{F}$. In this way, probability space is endowed with the structure of measurable space.

Finally, the third component of probability space is a probability measure $P$. It is a mapping of the set $\mathcal{F}$ onto the set of real numbers (each $F \in \mathcal{F}$ is mapped to a number $P(F)$ ), This mapping satisfies the following conditions: (a) $0 \leq P(F) \leq 1$ for any $F \in \mathcal{F}, P(\Omega)=1$; (b) $P\left(\sum_{j} F_{j}\right)=\sum_{j} P\left(F_{j}\right)$ for any countable family of disjoint subsets $F_{j} \in \mathcal{F}$.

We stress that probability measure is defined only for the events belonging to algebra $\mathcal{F}$. Generally, the probability may not be defined for elementary events.

Let us clarify the last statement with an example. Let the space of elementary events be the set of rational numbers lying between zero and unity. A trial is guessing of a number given by an interlocutor. Evidently, the probability of guessing of a number cannot have any value other than zero. However, it also cannot be zero. Indeed, any given number is with unit probability between zero and unit. The set of rational numbers is countable. Thus, by the properties of probability measure, a unit should be a countable sum of zeros. Contradiction is avoided if we take as $\mathcal{F}$ the set of all intervals (and their unions), and set the probability of each interval to its length.

We see that measurability is a crucial property of probability space. We will see below that it is even more important in the quantum case. Also, measurability is needed not only for mathematical consistency; it carries an important physical meaning.

Let us discuss now how the basic principles of probability theory are applied to quantum measurements. Most quantum measurements are related to obtaining probability distributions of some observables. With particular measuring devices we can obtain such distribution for a collection of compatible observables. In terms of probability theory, choosing a certain measuring instrument we chose a specific $\sigma$-algebra $\mathcal{F}$.

For clarity, we continue the discussion with an example. Let the system under study be a particle that moves in a fixed plane. Let us first look for a probability distribution of coordinate $X$ of the particle. Aiming at this, we split the plane into strips perpendicular to axis $X$. The width of the strips should be adjusted to the sensitivity of the measuring device. The strips will be elements $F_{i}^{X}$ of the algebra $\mathcal{F}_{X}$. With the measuring device we can determine the probability for 
the particle to be detected in a particular strip. Similar experiment can be performed to determine the probability distribution over the axis $Y$. In this case, the strips will be denoted as $F_{j}^{Y}$, and the $\sigma$-algebra as $\mathcal{F}_{Y}$.

We can perform a more detailed study and obtain the probability distribution in both coordinates simultaneously. To this end, we are to split the plane into rectangles resulting from intersections of various strips: $F_{i j}^{X Y}=F_{i}^{X} \cap F_{j}^{Y}$. The rectangles $F_{i j}^{X Y}$ will be the elements of $\sigma$-algebra $\mathcal{F}_{X Y}$. Algebra $\mathcal{F}_{X Y}$ is referred to as being generated by the algebras $\mathcal{F}_{X}$ and $\mathcal{F}_{Y}$. Until now, there was no difference between classical and quantum considerations.

Suppose now that we are looking for probability distributions not only for coordinates but also for momenta. If we are interested in distributions over coordinates and separately over momenta, the experiment can be organized as before. The only difference is that the strips should now be in the plane of momenta.

The situation is drastically different if we try to determine the combined probability distribution over the $X$ th coordinate and $K_{x}$ th projection of the momentum. Formally, in mathematics (see, e.g., [25]), we can construct a $\sigma$-algebra $\mathcal{F}_{X K_{x}}$ generated by the algebras $\mathcal{F}_{X} \mathcal{F}_{K_{x}}$. The elements of this algebra are rectangles (and various unions of the rectangles) in the two-dimensional plane $\left(X K_{x}\right)$ of the four-dimensional phase space. In the classical case, we can perform an experiment to determine the probability of detecting a particle in such a rectangle. However, in the quantum case, such an experiment is not possible because the devices measuring the $X$ th coordinate and $K_{x}$ th projection of the momentum are not compatible. This implies that it is not possible to ascribe a probability measure to such a rectangle. In other words, there is no notion of probability for an event of detecting a particle in such a rectangle.

From this example, we draw the following general conclusion. Not any mathematically possible (and classically allowable) $\sigma$-algebra is allowable as the $\sigma$-algebra of probability space in quantum space.

We conclude that experimentally an element of the measurable space $(\Omega, \mathcal{F})$ corresponds to a pair of the object under study and a particular type of measuring device. This type of device allows one to register an event corresponding to a set of compatible measurable quantities, i.e., corresponding to a fixed algebra $\mathfrak{Q}_{\xi}$. In view of this, $\sigma$-algebra $\mathcal{F}$ can also be labeled with parameter $\xi: \mathcal{F}=\mathcal{F}_{\xi}$.

The specifics of quantum experiments require particular care in defining one of the basic notions of probability theory, the real random quantity. Customarily, the real random quantity is defined as a mapping of space $\Omega$ of elementary events onto the extended real axis $\mathcal{R}=[-\infty,+\infty]$. However, this definition does not take into account the specifics of the quantum experiment, where the outcome may depend on the type of measuring device. In view of this, we take the following extended definition.

Definition 42. A real random quantity is a mapping of a measurable space $\left(\Omega, \mathcal{F}_{\xi}\right)$ of elementary events onto the extended real axis.

For observable $\hat{A}$ it can be expressed as follows:

$$
\varphi \stackrel{\hat{A}}{\longrightarrow} A_{\xi}(\varphi) \equiv \varphi_{\xi}(\hat{A}) \in \mathcal{R} .
$$

We call the quantum ensemble a set of identical physical systems (this means that they share the set $\mathfrak{A}_{+}$of observables, and the set $\left\{\mathfrak{Q}_{\xi}\right\}$ of commutative algebra $\mathfrak{Q}_{\xi}(\xi \in \Xi)$ ) that are in some quantum state. A quantum ensemble mixture that involves each of these ensembles with multiplicity $C_{i}\left(C_{i} \geq 0, \quad \sum C_{i}<\infty\right)$ is called a mixed ensemble. Experiment testifies in favor of the following postulate. 
Postulate 5. A quantum (generally, mixed) ensemble can be equipped with the structure of probability space. A reproducible measurement transforms a quantum ensemble into a new quantum ensemble that may have a new probability distribution of observables.

Consider an ensemble of physical systems occupying the quantum state $\Psi_{\varphi \eta}(\eta \in \Xi)$. In this case, we consider the equivalence class $\{\varphi\}_{\varphi \eta}$ as the space $\Omega\left(\varphi_{\eta}\right)$ of elementary even $\varphi$. Let a value of observable $\hat{A} \in \mathfrak{Q}_{\xi}$ be measured in an experiment employing a device of type $\xi$. We denote as $\left(\Omega\left(\varphi_{\eta}\right), \mathcal{F}_{\xi}\right)$ the corresponding measurable space. Let $P_{\xi}$ be the probability measure on this space; i.e., $P_{\xi}(F)$ is the probability of event $F \in \mathcal{F}_{\xi}$.

We take that event $F_{A}$ is realized in an experiment if the detected value of observable $\hat{A}$ does not exceed $A$. The probability of this event is $P_{\xi}\left(F_{A}\right)=P\left(\varphi: \varphi_{\xi}(\hat{A}) \leq A\right)$. Knowing the probabilities $P_{\xi}(F)$ suffices to obtain via summations and integrations the probability $P_{\xi}\left(F_{A}\right)$; distribution $P_{\xi}\left(F_{A}\right)$ is marginal with respect to the probabilities $P_{\xi}(F)$ (see, e.g., [26]).

Observable $\hat{A}$ may belong, apart from the algebra $\mathfrak{Q}_{\xi}$, to another maximal algebra $\mathfrak{Q}_{\xi^{\prime}}$. If so, we can use a device of type $\xi^{\prime}$ to measure the probability of the event $F_{A}$. In this case, another value $P_{\xi^{\prime}}\left(F_{A}\right)$ could be obtained for the probability. However, experiment shows that the probabilities do not depend on the measuring device in use. Thus, we should adopt one more postulate.

Postulate 6. If observable $\hat{A} \in \mathfrak{Q}_{\xi} \cap \mathfrak{Q}_{\xi^{\prime}}$ the probability of detecting event $F_{A}$ for a system in quantum state $\Psi_{\varphi \eta}$ does not depend on the type of measuring device, i.e., $\left.P\left(\varphi: \varphi_{\xi}(\hat{A})\right) \leq A\right)=$ $P\left(\varphi: \varphi_{\xi^{\prime}}(\hat{A}) \leq A\right)$.

Thus, despite the fact that elementary state $\varphi$ is a set of functional $\varphi_{\xi}$, we can use the notation $P(\varphi: \varphi(\hat{A}) \leq A)$ for the probability of event $F_{A}$.

Let us also introduce the following notation:

$$
P_{\hat{A}}(d \varphi)=P(\varphi: \varphi(\hat{A}) \leq A+d A)-P(\varphi: \varphi(\hat{A}) \leq A)
$$

and consider the ensemble of quantum systems occupying the quantum state $\Psi_{\varphi \eta}$. By probability theory (see, e.g., [25]) the expectation value of observable $\hat{A}$ in this state is given by the expression

$$
\langle\hat{A}\rangle=\int_{\varphi \in \Psi_{\varphi \eta}} P_{\hat{A}}(d \varphi) A(\varphi) \equiv \int_{\varphi \in \Psi \varphi \eta} P_{\hat{A}}(d \varphi) \varphi(\hat{A}) .
$$

On the other hand, the Khinchin theorem holds (see the law of large numbers, for example, in $[25]$ :

TheOREM. Let $A_{i}=\varphi_{i}(\hat{A}) \quad\left(1 \leq i \leq n, \quad \varphi_{i} \in \Psi_{\varphi \eta}\right)$ be a sequence of mutually independent randomly selected quantities with one and the same probability distribution having a finite expectation value $\langle\hat{A}\rangle$. Then the quantity $\left(A_{1}+\ldots+A_{n}\right) / n$ converges in probability to $\langle\hat{A}\rangle$ at $n \rightarrow \infty$. Thus,

$$
\Psi_{\varphi \eta}(\hat{A}) \equiv \lim _{n \rightarrow \infty} \mathrm{P}\left[n^{-1}\left(\varphi_{1}(\hat{A})+\ldots+\varphi_{n}(\hat{A})\right)\right]=\langle\hat{A}\rangle .
$$

Equation (5) defines a functional (the quantum average) on the set $\mathfrak{A}_{+}$. We denote this functional as $\Psi \varphi \eta(\cdot)$, and call it also the quantum state. Equation (5) and the properties of functionals $\varphi_{i}(\cdot)$ imply immediately that $\Psi \varphi \eta(\cdot)$ is linear on each subset $\mathfrak{Q}_{\xi}$ of compatible observables. In other words, restriction of functional $\Psi \varphi \eta(\cdot)$ onto each subset $\mathfrak{Q}_{\xi}$ is a linear functional. The linearity property of functional $\Psi \varphi \eta(\cdot)$ can be extended on the whole set $\mathfrak{A}_{+}$. Before this, the set $\mathfrak{A}_{+}$should be equipped with the structure of real linear space.

Since any element $\hat{A}$ of the set $\mathfrak{A}_{+}$belongs to some linear space $\mathfrak{Q}_{\xi}$, its multiplication by real numbers is defined. The addition of elements $\hat{A}$ and $\hat{B}$ is more involved, because these elements 
may belong to different linear spaces $\mathfrak{Q}_{\xi}$ and $\mathfrak{Q}_{\xi^{\prime}}$. However, the totality of quantum experiments points to the following fact. For any $\hat{A}$ and $\hat{B}$ belonging to $\mathfrak{A}_{+}$there exists such $\hat{D} \in \mathfrak{A}_{+}$that for any quantum state $\Psi \varphi \eta(\cdot)$ we have

$$
\Psi \varphi \eta(\hat{A})+\Psi \varphi \eta(\hat{B})=\Psi \varphi \eta(\hat{D}) .
$$

This element $\hat{D}$ can be taken by definition to be the sum of elements $\hat{A}$ and $\hat{B}$, i.e., $\hat{D}=\hat{A}+\hat{B}$. In view of these considerations, we accept the following postulate.

Postulate 7 . The set $\mathfrak{A}_{+}$can be equipped with the structure of a real vector space, and functionals $\Psi \varphi \eta(\cdot)$ are linear on that space.

This means that

$$
\Psi \varphi \eta(\hat{A})+\Psi \varphi \eta(\hat{B})=\Psi \varphi \eta(\hat{A}+\hat{B})
$$

This relation also holds when $\hat{A}$ and $\hat{B}$ belong to different subsets $\mathfrak{Q}_{\xi}$ and $\mathfrak{Q}_{\xi^{\prime}}$.

The set $\mathfrak{A}_{+}$can be equipped with the structure of real algebra. The product is defined as follows:

$$
\hat{A} \circ \hat{B}=1 / 2\left((\hat{A}+\hat{B})^{2}-\hat{A}^{2}-\hat{B}^{2}\right) .
$$

This product is obviously commutative, but may be nonassociative (see (D.3)), i.e., associator $\{\hat{A}, \hat{B}, \hat{D}\}=(\hat{A} \circ \hat{B}) \circ \hat{D}-\hat{A} \circ(\hat{B} \circ \hat{D})$ is not necessarily zero. It can be demonstrated (see [6]) that vanishing of the associator $\{\hat{A}, \hat{B}, \alpha \hat{I}\}$ for any $\hat{A}$ and $\hat{B}$ and any real number $\alpha$ is necessary and sufficient for distributivity (see D.2.b,c) of the product $\hat{A} \circ \hat{B}$. Under this condition, the real algebra with the product (6) is called real Jordan algebra [27, 6].

In principle, it is possible to base quantum theory on this algebra. Success has been very limited in this direction (see [6]). Much more successful has been the approach based on a complex associative algebra, which, in a certain sense, contains the Jordan algebra as it real part.

All Jordan algebras are classified into special and exceptional. The special algebras are defined as follows. Consider real or complex algebra $\mathfrak{A}$ with a "conventional" product $\hat{U} \hat{V} \quad(\hat{U} \in$ $\mathfrak{A}, \hat{V} \in \mathfrak{A}, \hat{U} \hat{V} \in \mathfrak{A})$. The algebra is associative with respect to this product, but not necessarily commutative. One can introduce the "symmetrized" product for the set $\mathfrak{A}$

$$
\hat{U} \circ \hat{V}=1 / 2(\hat{U} \hat{V}+\hat{V} \hat{U})
$$

With respect to this product, the set $\mathfrak{A}$ is a Jordan algebra. Any Jordan algebra isomorphic to such an algebra (or to its subalgebra) is called a special algebra. Otherwise, a Jordan algebra is called exceptional. Not any Jordan algebra is special. Thus, to be special, a Jordan algebra should imply some identities between its elements. In principle, such identities could be checked in experiments. However, the list of these identities is presently not known. On the other hand, in all quantum mechanical models considered until now the set of observables can be equipped with the structure of a special Jordan algebra. We stay within this tradition and accept the following hypothesis.

Hypothesis. There exists an involutive, associative, probably noncommutative algebra $\mathfrak{A}$ satisfying the following conditions:

(a) for any element $\hat{U} \in \mathfrak{A}$ there exists a Hermitian element $\hat{A}$ such that $\hat{U}^{*} \hat{U}=\hat{A}^{2}$;

(b) $\hat{U}^{*} \hat{U}=0$ implies that $\hat{U}=0$;

(c) the set of Hermitian elements of the algebra $\mathfrak{A}$ coincides with the set $\mathfrak{A}_{+}$of the observables.

Hereafter, elements of algebra $\mathfrak{A}$ are called dynamical quantities.

Evidently, set $\mathfrak{A}_{+}$can be equipped with the structure of Jordan algebra defining the product of its elements with equation (7). This hypothesis means that the Jordan algebra of observables 
is special and real. Correspondingly, dynamical quantities can be added and multiplied using the usual rules of addition and multiplication (apart from commutation). This seems to be so evident that it is almost never discussed. Despite this, we call the corresponding statements a hypothesis, not a postulate, because we cannot point out an experimental means of checking the necessity of these statements.

We stress that in the standard quantum mechanics the statements of the hypothesis are accepted in a much stronger form. Apart from our assumptions, it is assumed there that the observables are self-adjoint operators in a Hilbert space. These extra assumptions are hardly self-evident.

Hereafter, we consider a physical system to be given if the algebra $\mathfrak{A}$ of its dynamic quantities is given. By the first postulate, the algebras $\mathfrak{Q}_{\xi}$ of compatible observables are the maximal real commutative subalgebras of algebra $\mathfrak{A}$ belonging to $\mathfrak{A}_{+}$. This implies in turn that the compatible observables are mutually commutative elements of algebra $\mathfrak{A}$ and the incompatible observables do not commute with each other.

We mentioned above that in the quantum case, the set $\Xi$ of subalgebras $\mathfrak{Q}_{\xi} \quad(\xi \in \Xi)$ has cardinality of continuum. Indeed, even if algebra $\mathfrak{A}$ is an algebra with only two noncommuting generators $\hat{A}_{1}$ and $\hat{A}_{2}$, the commutative algebra $\mathfrak{Q}_{\alpha}$ with the generator $\hat{A}_{\alpha}=\hat{A}_{1} \cos \alpha+\hat{A}_{2} \sin \alpha$ will be an algebra of type $\mathfrak{Q}_{\xi}$ at any real $\alpha$.

\section{$5 \quad C^{*}$-ALGEBRA AND HILBERT SPACE}

Any element $\hat{U}$ of algebra $\mathfrak{A}$ can be uniquely represented in the form $\hat{U}=\hat{A}+i \hat{B}$, where $\hat{A}, \hat{B} \in$ $\mathfrak{A}_{+}$. Therefore, functional $\Psi_{\varphi \eta}(\cdot)$ can be uniquely extended to a linear functional on algebra $\mathfrak{A}$ : $\Psi_{\varphi \eta}(\hat{U})=\Psi_{\varphi \eta}(\hat{A})+i \Psi_{\varphi \eta}(\hat{B})$.

Let us define a semi-norm of element $\hat{U}$ with the

$$
\|\hat{U}\|^{2}=\sup _{\xi} \sup _{\varphi_{\xi}} \varphi_{\xi}\left(\hat{U}^{*} \hat{U}\right)=r\left(\hat{U}^{*} \hat{U}\right)
$$

where $r\left(\hat{U}^{*} \hat{U}\right)$ is the spectral radius of the element $\hat{U}^{*} \hat{U}$ on algebra $\mathfrak{A}$.

This is an acceptable definition. First, $\|\hat{U}\|^{2} \geq 0$ due to the property (P.7.c). Next, due to definition of the probability measure, we have for any $\eta \in \Xi$ that

$$
\Psi_{\varphi \eta}\left(\hat{U}^{*} \hat{U}\right)=\int_{\varphi \in\{\varphi\} \varphi \eta} P_{\hat{U}^{*} \hat{U}}(d \varphi) \varphi\left(\hat{U}^{*} \hat{U}\right) \leq \sup _{\xi} \sup _{\varphi_{\xi}} \varphi_{\xi}\left(\hat{U}^{*} \hat{U}\right)=r\left(\hat{U}^{*} \hat{U}\right)
$$

Next, for such $\eta \in \Xi$ that $\hat{U}^{*} \hat{U} \in \mathfrak{Q}_{\eta}$ we have $\Psi_{\varphi \eta}\left(\hat{U}^{*} \hat{U}\right)=\varphi_{\eta}\left(\hat{U}^{*} \hat{U}\right)$. Thus, for such $\eta$

$$
\sup _{\varphi_{\eta}} \Psi_{\varphi \eta}\left(\hat{U}^{*} \hat{U}\right)=\sup _{\varphi_{\eta}} \varphi_{\eta}\left(\hat{U}^{*} \hat{U}\right)=r_{\eta}\left(\hat{U}^{*} \hat{U}\right)
$$

where $r_{\eta}\left(\hat{U}^{*} \hat{U}\right)$ is the spectral radius in $\mathfrak{Q}_{\eta}$ since the subalgebra $\mathfrak{Q}_{\eta}$ is maximal (see (P.2), $r_{\eta}\left(\hat{U}^{*} \hat{U}\right)=$ $r\left(\hat{U}^{*} \hat{U}\right)$. From this, using Eqs. (8), (9), and (10), we obtain

$$
\|\hat{U}\|^{2}=\sup _{\xi} \sup _{\varphi_{\xi}} \varphi_{\xi}\left(\hat{U}^{*} \hat{U}\right)=\sup _{\xi} \sup _{\varphi_{\xi}} \Psi_{\varphi \xi}\left(\hat{U}^{*} \hat{U}\right) .
$$

Since $\Psi_{\varphi \xi}(\cdot)$ is a linear positive functional, the Cauchy-Schwarz inequality holds (see (P.5.b)):

$$
\left|\Psi_{\varphi \xi}\left(\hat{U}^{*} \hat{V}\right) \Psi_{\varphi \xi}\left(\hat{V}^{*} \hat{U}\right)\right| \leq \Psi_{\varphi \xi}\left(\hat{U}^{*} \hat{U}\right) \Psi_{\varphi \xi}\left(\hat{V}^{*} \hat{V}\right) .
$$

This implies that $\|\hat{U}\|$ satisfies the semi-norm axioms for element $\hat{U}$ (see, e.g., [6]):

$$
\|\hat{U}+\hat{V}\| \leq\|\hat{U}\|+\|\hat{V}\|, \quad\|\lambda \hat{U}\|=|\lambda|\|\hat{U}\|, \quad\left\|\hat{U}^{*}\right\|=\|\hat{U}\|, \quad\|\hat{U} \hat{V}\| \leq\|\hat{U}\|\|\hat{V}\| .
$$


Let us consider now the set $\mathfrak{J}$ of elements $\hat{U}$ of algebra $\mathfrak{A}$ for which $\|\hat{U}\|^{2}=0$. Inequality (12) implies that $\mathfrak{J}$ is a two-sided ideal of $\mathfrak{A}$. Thus, we can form a factor algebra $\mathfrak{A}^{\prime}=\mathfrak{A} / \mathfrak{J}$. For algebra $\mathfrak{A}^{\prime},\|\hat{U}\|^{2}=0$ implies that $\hat{U}=0$. Thus, equality (8) defines a norm on algebra $\mathfrak{A}^{\prime}$. On the other hand, it can be checked that algebra $\mathfrak{A}^{\prime}$ contains the same physical information as $\mathfrak{A}$ does.

For this check, we consider two observables $\hat{A}$ and $\hat{B}$ that either simultaneously belong or simultaneously do not belong to each of the subalgebras $\mathfrak{Q}_{\xi}$. Let $\hat{A}$ and $\hat{B}$ satisfy the extra condition $\|\hat{A}-\hat{B}\|=0$. Then equation (8) implies that

$$
\varphi_{\xi}(\hat{A})=\varphi_{\xi}(\hat{B})
$$

for all $\mathfrak{Q}_{\xi}$ containing these observables. Equality (13) means that no experiment can tell the difference between these observables. Thus, from the phenomenological standpoint, these observables should be considered as identical. Mathematically, these observables are equivalent modulo the ideal $\mathfrak{J}$. Under the transition from algebra $\mathfrak{A}$ to algebra $\mathfrak{A}^{\prime}$, all the equivalent observables are mathematically equated. Making a shortcut to algebra $\mathfrak{A}^{\prime}$, we accept the following postulate.

Postulate 8. If $\sup _{\xi} \sup _{\varphi_{\xi}}\left|\varphi_{\xi}(\hat{A}-\hat{B})\right|=0, \hat{A}=\hat{B}$.

This postulate is of a technical character. At the same time, it does not pose any restrictions from the phenomenological standpoint. Its only role is to simplify the mathematical description of physical systems. Hereafter, we assume that Postulate 8 is fulfilled, and, therefore, Eq. (11) defines the norm of element $\hat{U}$.

The multiplicative properties of functional $\varphi_{\xi}$ imply that $\varphi_{\xi}\left(\left[\hat{U}^{*} \hat{U}\right]^{2}\right)=\left[\varphi_{\xi}\left(\hat{U}^{*} \hat{U}\right)\right]^{2}$. Therefore, $\left\|\hat{U}^{*} \hat{U}\right\|=\|\hat{U}\|^{2}$. Thus, completion of algebra $\mathfrak{A}$ by the norm $\|\cdot\|$ makes $\mathfrak{A}$ a $C^{*}$-algebra [15]. We conclude that the algebra of quantum dynamical quantities can be equipped with the structure of $C^{*}$-algebra. In the standard algebraic approach to quantum theory, this statement plays the role of a basic axiom. Mathematically, it is very convenient. However, phenomenologically, the necessity of this axiom was unclear.

In most of our previous constructions, elementary state $\varphi=\left[\varphi_{\xi}\right]$ was a basic ingredient. The elementary state possesses many of the properties usually ascribed to the so-called hidden parameters [28]. Since the times of von Neumann [1], there is a strong opinion in standard quantum mechanics that hidden parameters cannot exist in quantum mechanics. In view of this, it is necessary to verify that elementary states can be introduced without contradictions.

Setting a physical system assumes setting an algebra $\mathfrak{A}$ of dynamical quantities. As we have argued above, this algebra should have the structure of $C^{*}$-algebra. Setting algebra $\mathfrak{A}$, we set at the same time the set of its maximal commutative associative subalgebras $\mathfrak{Q}_{\xi}$. Each of these subalgebras is a Banach algebra.

Clearly, constructing any elementary state $\varphi=\left[\varphi_{\xi}\right]$ is equivalent to constructing all its components $\varphi_{\xi}$, and each $\varphi_{\xi}$ is a character of the subalgebra $\mathfrak{Q}_{\xi}$. Each functional $\varphi_{\xi}$ can be constructed as follows. Select in an arbitrary way the system of independent generating elements $G\left(\mathfrak{Q}_{\xi}\right)$ in the subalgebra $\mathfrak{Q}_{\xi}$. Using (P.8), relate each element of the set $G\left(\mathfrak{Q}_{\xi}\right)$ to a point of its spectrum. In this way, we define the functional $\varphi_{\xi}$ on the set $G\left(\mathfrak{Q}_{\xi}\right)$. By linearity and multiplicativity, functional $\varphi_{\xi}$ can be extended unambiguously to the whole subalgebra $\mathfrak{Q}_{\xi}$. Sorting out all the points of the spectrum for each element of the set $G\left(\mathfrak{Q}_{\xi}\right)$, we construct all possible functionals $\varphi_{\xi}$. For another $\xi$, functionals $\varphi_{\xi}$ are constructed in the same way. This is a consistent procedure if the functionals are constructed independently for different $\xi$. If we impose the condition (3), the procedure may turn out, and sometimes does turn out self-contradictory.

However, it is always possible to construct an elementary state $\varphi$ that is stable on all the observables belonging to any single subalgebra $\mathfrak{Q}_{\xi}$. This is achieved if we start constructing the functional $\varphi$ from this very subalgebra using the above procedure. On another subalgebra $\mathfrak{Q}_{\xi^{\prime}}$, the functional $\varphi_{\xi^{\prime}}$ is constructed as follows. Let $\mathfrak{Q}_{\xi} \cap \mathfrak{Q}_{\xi^{\prime}}=\mathfrak{Q}_{\xi \xi^{\prime}}$, and let $G\left(\mathfrak{Q}_{\xi \xi^{\prime}}\right)$ be the independent 
generating elements of subalgebra $\mathfrak{Q}_{\xi \xi^{\prime}}$. Let $\tilde{G}\left(\mathfrak{Q}_{\xi \xi^{\prime}}\right)$ be a supplement of these generating elements to the set of generating elements of algebra $\mathfrak{Q}_{\xi^{\prime}}$. If $\hat{A} \in \mathfrak{Q}_{\xi \xi^{\prime}}$, set $\varphi_{\xi^{\prime}}(\hat{A})=\varphi_{\xi}(\hat{A})$. If $\hat{A} \in \tilde{G}\left(\mathfrak{Q}_{\xi \xi^{\prime}}\right)$, construct $\varphi_{\xi^{\prime}}(\hat{A})$ as a mapping of the element $\hat{A}$ to one of the points of its spectrum. Functional $\varphi_{\xi^{\prime}}$ is extended to another element of subalgebra $\mathfrak{Q}_{\xi^{\prime}}$ by linearity and multiplicativity.

We conclude that there is no problem of existence for elementary states.

The proof due to von Neumann [1] of the inexistence of hidden parameters is invalid for the elementary states $\varphi$ for the following reason. It is assumed in the proof that the state is described by a linear functional on the set of observables. Elementary state $\varphi$ can be considered as a functional on the set of observables. However, it is linear only on the subsets $\mathfrak{Q}_{\xi}$. Besides, it is a multivalued functional.

In the same proof, von Neumann demonstrated that linearity of the functional describing the state of the system is incompatible with the assumption on the presence of microcausality. He concluded that microcausality is absent, and macrocausality appears due to averaging over a large number of noncausal events. Similarly, Blokhintsev maintained the view [20, 22, 23] that causality is not a property of individual quantum objects, but characterizes only ensembles of such objects. It allows one to consider quantum processes governed by the Schrodinger equation as causal. In our approach we resolve the "linearity-causality" dilemma in the opposite way. Microscopically, causality is present, but there is no linearity of the state describing an individual quantum system. Linearity of a quantum state appears due to averaging over a quantum ensemble.

We point out that the appearance of linearity after averaging is a not an infrequent phenomenon in probability theory. Therefore, the principles of linearity and superposition considered as basic physical principles in the standard quantum mechanics are deprived of this status in reality. These properties are only mathematical artifacts appearing due to an averaging procedure. In contrast, causality is a physical principle, which is widely used in physics, ignoring the "official ban." Namely, an elementary state is able to claim the role of a mathematical image of the reality physically supporting causality.

The superposition property mentioned above originates from the following remarkable property of $C^{*}$-algebra. Any $C^{*}$-algebra is isometrically isomorphic to the subalgebra of linear bounded operators in a Hilbert space [15]. It will allow us later on to use the familiar formalism of Hilbert space, where the superposition property appears naturally.

REMARK. It is usually assumed in the standard quantum mechanics that all self-adjoint bounded operators in Hilbert space are observables. This assumption is violated in the models with superselection rules [29]. The algebraic approach (also, its version in this paper) does not need this assumption.

In the algebraic approach, a state is defined as a positive linear functional $\Psi$ on the set of observables satisfying the normalization condition $\Psi(\hat{I})=1$. In the standard quantum mechanics, a state is given either with a vector of Hilbert space, or, more generally, with a density matrix. However, not any physically interesting state can be given with a density matrix (see [6]). Because of this, the algebraic definition is more general. Frequently, a state defined in this way is called algebraic. Since $\varphi(\hat{I})=1$ (see (P.7.b)), the functional $\Psi_{\varphi \eta}(\hat{A})$ defined by formula (4) satisfies the normalization condition. Thus, the quantum state defined in this paper is an algebraic state. Since any linear positive functional defined on the set of observables can be unambiguously extended on the algebra of dynamical quantities, hereafter we call the algebraic state a linear positive normalized functional defined on algebra $\mathfrak{A}$.

Definition 43. Algebraic state $\Psi$ is called a pure state if the equality

$$
\Psi=\lambda \Psi_{1}+(1-\lambda) \Psi_{2} \quad 0<\lambda<1
$$


where $\Psi_{1}$ and $\Psi_{2}$ are two states, implies that $\Psi_{1}=\Psi_{2}$.

It is easily checked that the quantum state $\Psi_{\varphi \xi}$ introduced in definition (D.41) is a pure algebraic state. Indeed, assume that functional $\Psi_{\varphi \xi}$ can be represented in the form (14). Restrict the equality (14) to the subalgebra $\mathfrak{Q}_{\xi}$. On this subalgebra, i.e., for any $\hat{A} \in \mathfrak{Q}_{\xi}$, the equality $\varphi_{\xi}(\hat{A})=$ $\Psi_{\varphi \xi}(\hat{A})$ is valid. However, functional $\varphi_{\xi}(\cdot)$ is a character of subalgebra $\mathfrak{Q}_{\xi}$. Any character of a commutative algebra is a pure state (see [14]). Therefore, equation $\Psi_{\varphi \xi}(\hat{A})=\lambda \Psi_{1}(\hat{A})+(1-\lambda) \Psi_{2}(\hat{A})$ implies that $\Psi_{1}(\hat{A})=\Psi_{2}(\hat{A})=\Psi_{\varphi \xi}(\hat{A})=\varphi_{\xi}(\hat{A})$ for any $\hat{A} \in \mathfrak{Q}_{\xi}$. In particular,

$$
\Psi_{1}\left(\left[\hat{A}-\varphi_{\xi}(\hat{A})\right]^{2}\right)=\varphi_{\xi}\left(\left[\hat{A}-\varphi_{\xi}(\hat{A})\right]^{2}\right)=0 .
$$

This implies that

$$
\int_{\varphi \in \Psi_{1}} P_{\hat{A}}(d \varphi) \varphi\left(\left[\hat{A}-\varphi_{\xi}(\hat{A})\right]^{2}\right) \equiv \Psi_{1}\left(\left[\hat{A}-\varphi_{\xi}(\hat{A})\right]^{2}\right)=0 .
$$

for any $\hat{A} \in \mathfrak{Q}_{\xi}$. Thus, if $\varphi \in \Psi_{1}$, almost surely, $\varphi(\hat{A})=\varphi_{\xi}(\hat{A})$ for $\hat{A} \in \mathfrak{Q}_{\xi}$. This means that almost surely elementary states $\varphi \in \Psi_{1}$ form the equivalence class $\{\varphi\}_{\varphi \xi}$. From this, we obtain that

$$
\Psi_{1}(\hat{A})=\int_{\varphi \in \Psi_{1}} P_{\hat{A}}(d \varphi) \varphi(\hat{A})=\int_{\varphi \in \Psi_{\varphi \xi}} P_{\hat{A}}(d \varphi) \varphi(\hat{A})=\Psi_{\varphi \xi}(\hat{A})
$$

for any $\hat{A}$. For $\Psi_{2}(\hat{A})$, we have an analogous derivation, i.e., $\Psi_{1}(\hat{A})=\Psi_{2}(\hat{A})$.

There is a procedure that realizes the relation between $C^{*}$ algebra and Hilbert space. It is called the Gelfand-Naimark-Segal (GNS) canonical construction (see, e.g., [13, 6]). Below, we give a brief exposition.

Suppose that some $C^{*}$-algebra $\mathfrak{A}$ and a positive functional $\Psi_{0}$ on this algebra are given. Two elements $\hat{U}, \hat{U}^{\prime} \in \mathfrak{A}$ are taken to be equivalent if the equality $\Psi_{0}\left(\hat{W}^{*}\left(\hat{U}-\hat{U}^{\prime}\right)\right)=0$ holds for any $\hat{W} \in \mathfrak{A}$. Denote by $\Phi(\hat{U})$ the equivalence class containing element $\hat{U}$, and consider the set of all equivalence classes $\mathfrak{A}\left(\Psi_{0}\right)$ in $\mathfrak{A}$. The set $\mathfrak{A}\left(\Psi_{0}\right)$ is a linear space if we take $a \Phi(\hat{U})+b \Phi(\hat{V})=$ $\Phi(a \hat{U}+b \hat{V})$. The scalar product in $\mathfrak{A}\left(\Psi_{0}\right)$ is defined as follows:

$$
(\Phi(\hat{U}), \Phi(\hat{V}))=\Psi_{0}\left(\hat{U}^{*} \hat{V}\right)
$$

This scalar product generates in $\mathfrak{A}\left(\Psi_{0}\right)$ the norm $\|\Phi(\hat{U})\|=\left[\Psi_{0}\left(\hat{U}^{*} \hat{U}\right)\right]^{1 / 2}$. Completion by this norm make $\mathfrak{A}\left(\Psi_{0}\right)$ a Hilbert space. Each element $\hat{V}$ of algebra $\mathfrak{A}$ is uniquely represented in this space by a linear operator $\Pi(\hat{V})$ acting by the rule

$$
\Pi(\hat{V}) \Phi(\hat{U})=\Phi(\hat{V} \hat{U})
$$

In this way, the GNS construction allows one to construct a representation of any $C^{*}$-algebra. Let us recall which representations are possible.

Representations can be exact or inexact. For an exact representation, different elements of the algebra are represented by different operators in the Hilbert space.

Definition 44. Representation $\hat{V} \rightarrow \Pi(\hat{V})$ is called exact if $\Pi(\hat{V})=0$ implies $\hat{V}=0$.

A representation can be zero.

Definition 45. Representation $\hat{V} \rightarrow \Pi(\hat{V})$ is called zero if $\Pi(\hat{V})=0$ at any $\hat{V}$. 
Definition 46. Representation $\hat{V} \rightarrow \Pi(\hat{V})$ is a direct orthogonal sum, $\Pi(\hat{V})=\Pi_{1}(\hat{V}) \oplus \Pi_{2}(\hat{V})$, of two (or a larger number) representations if the operators of $\Pi(\hat{V})$ act in the Hilbert space $\mathfrak{H}=\mathfrak{H}_{1} \oplus \mathfrak{H}_{2}$ by the rule $\Pi(\hat{V}) \Phi=\Pi_{1}(\hat{V}) \Phi_{1}+\Pi_{2}(\hat{V}) \Phi_{2}$. Here, $\Phi=\Phi_{1}+\Phi_{2}, \quad \Phi_{1} \in \mathfrak{H}_{1}, \Phi_{2} \in \mathfrak{H}_{2}$, while $\Pi_{1}(\hat{V})$ and $\Pi_{2}(\hat{V})$ are the operators of representations in the spaces $\mathfrak{H}_{1}$ and $\mathfrak{H}_{2}$, respectively.

Definition 47. Representation $\hat{V} \rightarrow \Pi(\hat{V})$ is called degenerate if it is representable as a direct orthogonal sum of representations among which at least one is a zero representation.

Definition 48. Representation $\hat{V} \rightarrow \Pi(\hat{V})$ is called irreducible if it is not possible to represent it as a direct orthogonal sum of two other representations.

Definition 49. Representation $\hat{V} \rightarrow \Pi(\hat{V})$ acting in a Hilbert space $\mathfrak{H}$ is called cyclic if there exists a vector $\Phi$ (called the cyclic vector) such that the set of vectors $\Pi(\hat{V}) \Phi$ is everywhere dense in $\mathfrak{H}$.

The latter is equivalent to the requirement that $\Pi(\hat{V}) \Phi$ contains a basis of $\mathfrak{H}$.

Evidently, GNS construction yields a cyclic nondegenerate representation. It can be demonstrated that this representation is irreducible if and only if $\Psi_{0}$ is a pure state. Generally, this representation is not an exact one. However, there exists the so-called universal representation $\hat{V} \rightarrow \Pi_{u}(\hat{V})$. This representation is a direct sum of representations, $\Pi_{u}(\hat{V})=\bigoplus_{i} \Pi_{i}(\hat{V})$. Each of the representations $\hat{V} \rightarrow \Pi_{i}(\hat{V})$ is yielded by GNS construction with a state $\Psi_{i}$. Summation runs over all the algebraic states $\Psi_{i}$.

Any nondegenerate representation of a $C^{*}$-algebra is isomorphic to a subrepresentation of the universal representation. The universal representation is exact. This means that the algebra of elements $\hat{V}$ is isomorphic to the algebra of operators $\Pi_{u}(\hat{V})$. In other words, $C^{*}$-algebra $\mathfrak{A}$ is isomorphic to the subalgebra of bounded linear operators in the Hilbert space $\mathfrak{H}_{u}$. Any algebraic relation between elements of $\mathfrak{A}$ can be established via establishing corresponding relations between the operators realizing any exact representation of the algebra. The existence of the universal representation guarantees that there exists at least one such representation.

We mentioned above that the quantum states introduced in this paper are pure algebraic states. Now we demonstrate how to construct the functionals with the required properties. Let us first consider the case when the commutative algebra $\mathfrak{Q}_{\xi}$, defining the quantum state contains a onedimensional projector $\hat{p}_{0}$. The most accessible definition of the one-dimensional projector is as such element of the algebra that is represented in any exact representation with a projection operator onto one-dimensional subspace of the Hilbert space.

REMARK. In the standard quantum mechanics, it is usually assumed that any bounded selfadjoint operator corresponds to an observable. In this case, any maximal commutative subalgebra contains one-dimensional projectors. Conversely, each one-dimensional projector belongs to some commutative subalgebra. In this situation, the case under consideration is the most general one.

So, let $\hat{p}_{0} \in \mathfrak{Q}_{\xi}$. Consider an exact representation of algebra $\mathfrak{A}$. There exists a vector $\left|\Phi_{0}\right\rangle$ in the Hilbert space of this representation such that $\hat{p}_{0}\left|\Phi_{0}\right\rangle=\left|\Phi_{0}\right\rangle, \quad\left\langle\Phi_{0} \mid \Phi_{0}\right\rangle=1, \quad \hat{p}_{0}=\left|\Phi_{0}\right\rangle\left\langle\Phi_{0}\right|$. For arbitrary $\hat{B} \in \mathfrak{A}$, consider the combination $\hat{p}_{0} \hat{B} \hat{p}_{0}=\left|\Phi_{0}\right\rangle\left\langle\Phi_{0}|\hat{B}| \Phi_{0}\right\rangle\left\langle\Phi_{0}|\equiv \vartheta(\hat{B})| \Phi_{0}\right\rangle\left\langle\Phi_{0}\right|$, i.e.,

$$
\hat{p}_{0} \hat{B} \hat{p}_{0}=\vartheta(\hat{B}) \hat{p}_{0}
$$

Relation (17) is a relation between elements of algebra $\mathfrak{A}$. Thus, it is a feature of algebra $\mathfrak{A}$, and not a property of the representation. In particular, functional $\vartheta(\hat{B})$ is independent of the representation. It is easily seen that $\vartheta(\hat{B})$ is an algebraic state of algebra $\mathfrak{A}$. Its linearity is implied 
by the relation

$$
\vartheta(\hat{B}+\hat{D}) \hat{p}_{0}=\hat{p}_{0}(\hat{B}+\hat{D}) \hat{p}_{0}=[\vartheta(\hat{B})+\vartheta(\hat{D})] \hat{p}_{0} .
$$

Its positivity is a consequence of the relation $\hat{p}_{0} \hat{B}^{*} \hat{B} \hat{p}_{0}=\vartheta\left(\hat{B}^{*} \hat{B}\right) \hat{p}_{0}$. Since operators $\hat{p}_{0} \hat{B}^{*} \hat{B} \hat{p}_{0}$ and $\hat{p}_{0}$ are positive, $\vartheta\left(\hat{B}^{*} \hat{B}\right) \geq 0$. Finally, normalization is implied by the relation $\vartheta(\hat{I}) \hat{p}_{0}=$ $\hat{p}_{0} \hat{I} \hat{p}_{0}=\hat{p}_{0}$. In addition, restriction of functional $\vartheta(\cdot)$ onto the subalgebra $\mathfrak{Q}_{\xi}$ is a character of this subalgebra. Indeed, let $\hat{A} \in \mathfrak{Q}_{\xi} \hat{B} \in \mathfrak{Q}_{\xi}$. Then

$$
\vartheta(\hat{A} \hat{B}) \hat{p}_{0}=\hat{p}_{0} \hat{A} \hat{B} \hat{p}_{0}=\hat{p}_{0} \hat{A} \hat{p}_{0} \hat{p}_{0} \hat{B} \hat{p}_{0}=\vartheta(\hat{A}) \vartheta(\hat{B}) \hat{p}_{0}
$$

We conclude that functional $\vartheta(\cdot)$ has all the properties required in Postulate 7 from a quantum average. Apart from this, $\vartheta(\cdot)$ is positive and satisfies the normalization condition. These are precisely the conditions that any functional describing a quantum state should satisfy. Equality (17)) is of a purely algebraic nature. Therefore, the value $\vartheta(\hat{B})$ depends only on $\hat{p}_{0}$ (the quantum state) and on $\hat{B}$ considered as an element of algebra $\mathfrak{A}$. However, it does not depend on any particular commutative subalgebra ( $\hat{B}$ may belong to a number of such subalgebras). This means that functional $\vartheta(\cdot)$ satisfies Postulate 6.

Let us demonstrate now that the opposite statement is also valid. If a functional $\Psi_{\xi}^{0}(\cdot)$ corresponds to a quantum state $\Psi_{\xi}^{0}$ that satisfies the condition $\varphi_{\xi}\left(\hat{p}_{0}\right)=1$, then $\Psi_{\xi}^{0}(\cdot)=\vartheta(\cdot)$. Indeed, equality (4) implies that

$$
\Psi_{\xi}^{0}\left(\hat{p}_{0}\right)=\Psi_{\xi}^{0}(\hat{I})=1 .
$$

With the Cauchy-Schwarz inequality (see formula (12)), we obtain

$$
\left.\left|\Psi_{\xi}^{0}\left(\hat{B}\left(\hat{I}-\hat{p}_{0}\right)\right)\right|^{2} \leq \Psi_{\xi}^{0} B^{*} \hat{B}\right) \Psi_{\xi}^{0}\left(\hat{I}-\hat{p}_{0}\right)
$$

From this, in view of equality (18), we have

$$
\Psi_{\xi}^{0}(\hat{B})=\Psi_{\xi}^{0}\left(\hat{B} \hat{p}_{0}\right)=\Psi_{\xi}^{0}\left(\hat{p}_{0} \hat{B}\right)
$$

Substitution $\hat{B} \rightarrow\left(\hat{I}-\hat{p}_{0}\right) \hat{B}$ in (19) yields

$$
\Psi_{\xi}^{0}(\hat{B})=\Psi_{\xi}^{0}\left(\hat{p}_{0} \hat{B} \hat{p}_{0}\right) .
$$

Using (17) in the right-hand-side of (20), we obtain the equality

$$
\Psi_{\xi}^{0}(\hat{B})=\Psi_{\xi}^{0}\left(\vartheta(\hat{B}) \hat{p}_{0}\right)=\vartheta(\hat{B}) .
$$

Consider now a GNS construction that uses $\Psi_{\xi}^{0}(\hat{B})$ as its generating functional. Let $\Phi_{0}(\hat{I})$ be the equivalence class of the element $\hat{I}$. Then, due to Eqs. (15) and (16), we have

$$
\left(\Phi_{0}(\hat{I}), \Pi(\hat{B}) \Phi_{0}(\hat{I})\right)=\Psi_{\xi}^{0}(\hat{B})
$$

for any $\hat{B} \in \mathfrak{A}$.

According to equalities (44) and (5) , functional $\Psi_{\xi}^{0}(\hat{B})$ describes the average value of the observable $\hat{B}$ in the quantum state $\Psi_{\xi}^{0}$. Equality (22) tells that this average coincides with the expectation value of the operator $\Pi(\hat{B})$ over the state described by vector $\Phi_{0}(\hat{I})$ of the Hilbert space. This observation allows one to employ to full extent the mathematical formalism of the standard quantum mechanics for computing quantum averages.

At the same time, there is a considerable difference between the present approach and standard quantum mechanics. In the latter, relations similar to (22) are postulated (the Born postulate [30]). This postulate suffices for computations in quantum mechanics, but its necessity remains unclear. 
In contrast, in our approach, equality (22) is a consequence of phenomenologically necessary postulates.

Let us pass on to the case when the subalgebra $\mathfrak{Q}_{\xi}$ does not contain one-dimensional projectors. In this case, an exact representation of algebra $\mathfrak{A}$ should be considered. Let $\mathfrak{H}$ be the Hilbert space of this representation and $\mathfrak{B}(\mathfrak{H})$ be the set of all bounded linear operators in $\mathfrak{H}$. Algebras $\mathfrak{A}$ and $\mathfrak{Q}_{\xi}$ may be considered as subalgebras of algebra $\mathfrak{B}(\mathfrak{H})$.

Let $\mathfrak{Q}_{\xi}^{\prime}$ be the maximal real commutative subalgebra of algebra $\mathfrak{B}(\mathfrak{H})$ such that $\mathfrak{Q}_{\xi}^{\prime} \supset \mathfrak{Q}_{\xi}$. Consider the set of all projectors belonging $\mathfrak{Q}_{\xi}^{\prime}$. These projectors are mutually commuting selfadjoint operators in $\mathfrak{H}$ having discrete spectra. There exists an orthonormal basis in $\mathfrak{H}$ consisting of the eigenvectors of these operators. Let $\{\hat{p}\}$ be the set of projectors onto such basis vectors. All these projectors are one-dimensional. They belong to $\mathfrak{B}(\mathfrak{H})$, but do not belong in the case under consideration to $\mathfrak{Q}_{\xi}$. Each of the projectors $\hat{p}_{i} \in\{\hat{p}\}$ defines a linear functional $\vartheta_{i}(\cdot) \mathfrak{B}(\mathfrak{H})$ : $\hat{p}_{i} \hat{A} \hat{p}_{i}=\vartheta_{i}(\hat{A}) \hat{p}_{i}$. Restriction of this functional onto the algebra $\mathfrak{A}$ has all the properties required for description of the corresponding pure quantum state.

\section{ILLUSTRATIONS}

Let us consider three simple examples illustrating the general considerations.

The first example is that of a two-level quantum system, whose observables are described with Hermitian matrixes $2 \times 2$. The algebra $\mathfrak{A}$ of dynamical quantities is in this case the set of all matrixes of the form

$$
\hat{A}=\left[\begin{array}{ll}
a & b \\
c & d
\end{array}\right],
$$

with the algebraic operations coinciding with the corresponding matrix operations.

It is easy to construct all the elementary states for such a system. Let $\hat{A}$ be a Hermitian matrix, i.e., $a^{*}=a, d^{*}=d, c=b^{*}$. Any such matrix is representable in the form

$$
\hat{A}=r_{0} \hat{I}+r \hat{\tau}(\boldsymbol{\xi}) .
$$

Here, $\boldsymbol{\xi}$ is a unit three-dimensional vector and $\tau_{i}$ are the Pauli matrixes, $\hat{\tau}(\boldsymbol{\xi})=(\boldsymbol{\tau} \cdot \boldsymbol{\xi})$. Equation (23) holds if

$$
\begin{gathered}
r=\left((a-d)^{2} / 4+b b^{*}\right)^{1 / 2}, \quad r_{0}=(a+d) / 2, \\
\xi_{1}=\left(b+b^{*}\right) /(2 r), \quad \xi_{2}=\left(b-b^{*}\right) /(2 i r), \quad \xi_{3}=(a-d) /(2 r) .
\end{gathered}
$$

Evidently, $\hat{\tau}(-\boldsymbol{\xi})=-\hat{\tau}(\boldsymbol{\xi})$. If $\boldsymbol{\xi}^{\prime} \neq \pm \boldsymbol{\xi}$, the commutator of the matrixes $\hat{\tau}(\boldsymbol{\xi})$ and $\hat{\tau}\left(\boldsymbol{\xi}^{\prime}\right)$ is nonvanishing. Thus, each $\hat{\tau}(\boldsymbol{\xi})$ (up to a sign) is a generating element of a real maximal commutative subalgebra $\mathfrak{Q}_{\boldsymbol{\xi}}$. Since $\hat{\tau}(\boldsymbol{\xi}) \hat{\tau}(\boldsymbol{\xi})=\hat{I}$, the spectrum of element $\hat{\tau}(\boldsymbol{\xi})$ consists of two points, \pm 1 .

Let $\varphi^{\alpha}=\left[\varphi_{\boldsymbol{\xi}}^{\alpha}\right]$ be an elementary state. Here, $\varphi_{\boldsymbol{\xi}}^{\alpha}$ is a character of subalgebra $\mathfrak{Q}_{\boldsymbol{\xi}}$, and the superscript $\alpha$ marks different elementary states. Consider a function $f^{\alpha}(\boldsymbol{\xi})$ satisfying the restriction $f^{\alpha}(-\boldsymbol{\xi})=-f^{\alpha}(\boldsymbol{\xi})$ and, for each $\boldsymbol{\xi}$, the value of the function is either +1 , or -1 ; superscript $\alpha$ marks different functions. Evidently, we can put $\varphi_{\boldsymbol{\xi}}^{\alpha}(\hat{\tau}(\boldsymbol{\xi}))=f^{\alpha}(\boldsymbol{\xi})$. Taking into account that $\hat{\tau}(\boldsymbol{\xi})$ is a generating element of subalgebra $\mathfrak{Q}_{\boldsymbol{\xi}}$, we obtain that

$$
\varphi_{\boldsymbol{\xi}}^{\alpha}(\hat{A})=r_{0}(\hat{A})+r(\hat{A}) f^{\alpha}(\boldsymbol{\xi})
$$

for any $\hat{A} \in \mathfrak{Q}_{\xi}$.

We point out that any observable $\hat{A}$ (which is not a multiple $\hat{I}$ ) belongs for this quantum system to one and only one maximal subalgebra $\mathfrak{Q}_{\boldsymbol{\xi}}$. Generally, this property certainly does not hold. Due 
to this peculiarity of the system under consideration, we can represent any elementary state as a whole functional defined on the whole set $\mathfrak{A}_{+}$:

$$
\varphi^{\alpha}(\hat{A})=r_{0}(\hat{A})+r(\hat{A}) f^{\alpha}(\boldsymbol{\xi}(\hat{A}))
$$

for any observable $\hat{A}$. Here, $r, r_{0}$, and $\boldsymbol{\xi}$ should be considered as functions of $\hat{A}$ (see formula (24)). This functional can be naturally extended onto the entire algebra $\mathfrak{A}$. This example gives extra evidence against the possibility of extending the proof of von Neumann for the inexistence of hidden parameters to elementary states.

Observe that functional $\varphi^{\alpha}(\hat{A})$ defined by formula (25) is nonlinear. Generally, an elementary state can be formally represented as a nonlinear functional defined on the whole algebra $\mathfrak{A}$. However, in the general case, this functional is not single valued, because one and the same observable $\hat{A}$ may simultaneously belong to several maximal commutative subalgebras $\mathfrak{Q}_{\xi}$.

Let us come back to the two-level system. The Hamiltonian of this system can be represented as

$$
\hat{H}=\left[\begin{array}{cc}
E_{0} & 0 \\
0 & -E_{0}
\end{array}\right]
$$

and, the projector onto the ground state, as

$$
\hat{p}_{0}=\left[\begin{array}{ll}
0 & 0 \\
0 & 1
\end{array}\right] \text {. }
$$

Evidently, $\hat{p}_{0} \hat{H} \hat{p}_{0}=-E_{0} \hat{p}_{0}, \hat{p}_{0} \Psi_{0}(\hat{A})=\hat{p}_{0} \hat{A} \hat{p}_{0}=\hat{p}_{0} d(\hat{A})$. Thus, the ground state is the linear functional $\Psi_{0}(\hat{A})=d(\hat{A})$. On the other hand, the ground state is the equivalence class of the elementary states determined by the condition $\varphi^{\alpha=0}\left(\hat{p}_{0}\right)=1$. From this condition we obtain that

$$
f^{0}\left(\xi_{1}=0, \xi_{2}=0, \xi_{3}=1\right)=-1 .
$$

We conclude that the ground state is the set of elementary states (25) that have function $f^{0}$ satisfying condition (26).

It is interesting to look at the appearance of the ergodicity property. We assume that the time evolution of the observables is defined in the usual way with the unitary automorphism

$$
\hat{A} \stackrel{t}{\longrightarrow} \hat{A}(t)=\exp (-i \hat{H} t) \hat{A} \exp (i \hat{H} t)
$$

and consider the observable $\tilde{A}$,

$$
\tilde{A}=\lim _{L \rightarrow \infty} \frac{1}{2 L} \int_{-L}^{L} d t \hat{A}(t)
$$

The limit and the integral are understood in the sense of the weak topology (see (D.33)). The spectral decomposition $\hat{H}=E_{0} \hat{p}_{1}-E_{0} \hat{p}_{0}$ is valid for $\hat{H}$, where $\hat{p}_{1}=\hat{I}-\hat{p}_{0}$. Thus, observable $\tilde{A}$ can be represented as follows:

$$
\tilde{A}=\lim _{L \rightarrow \infty} \frac{1}{2 L} \int_{-L}^{L} d t\left[\hat{p}_{0} \hat{A} \hat{p}_{0}+\hat{p}_{1} \hat{A} \hat{p}_{1}+\hat{p}_{0} \hat{A} \hat{p}_{1} e^{2 i E_{0} t}+\hat{p}_{1} \hat{A} \hat{p}_{0} e^{-2 i E_{0} t}\right]=\hat{p}_{0} \hat{A} \hat{p}_{0}+\hat{p}_{1} \hat{A} \hat{p}_{1} .
$$

Let $\varphi^{0}$ be an elementary state belonging to the ground quantum state. Then,

$$
\varphi^{0}\left(\hat{p}_{1}\right)=\varphi^{0}\left(\hat{I}-\hat{p}_{0}\right)=0
$$

Since $\left[\hat{p}_{0} \hat{A} \hat{p}_{0}, \hat{p}_{1} \hat{A} \hat{p}_{1}\right]=0$,

$$
\varphi^{0}(\tilde{A})=\varphi^{0}\left(\hat{p}_{0} \hat{A} \hat{p}_{0}\right)+\varphi^{0}\left(\hat{p}_{1} \hat{A} \hat{p}_{1}\right)=\Psi_{0}(\hat{A})+\varphi^{0}\left(\hat{p}_{1}\right) \varphi^{0}\left(\hat{p}_{1} \hat{A} \hat{p}_{1}\right) .
$$


This relation implies that

$$
\varphi^{0}(\tilde{A})=\Psi_{0}(\hat{A}) .
$$

We conclude that the value of the observable $\hat{A}$ averaged over the ensemble described by the ground quantum state equals the value this observable averaged over time in any elementary state belonging to the ground quantum state.

Our second example is the one-dimensional harmonic oscillator. We are interested in the Green's functions of this system. Surely, it is possible to pass on to the standard scheme that uses the Hilbert space via the GNS construction. However, it is possible to suggest a method that is more straightforward from the standpoint of our approach.

We take that a harmonic oscillator is a physical system described with an algebra $\mathfrak{A}$. of dynamical quantities. This is the algebra with two Hermitian generating elements $\hat{X}$ and $\hat{K}$ satisfying the commutation relation

$$
[\hat{X}, \hat{K}]=i \text {. }
$$

We use the natural units where $\hbar=m=1$. The time evolution in algebra $\mathfrak{A}$ is governed with the Hamiltonian $\hat{H}=1 / 2\left(\hat{K}^{2}+\omega^{2} \hat{X}^{2}\right)$. Elements $\hat{X}, \hat{K}$, and $\hat{H}$ are unbounded, and, therefore, they do not belong to $C^{*}$-algebra. However, it is possible to use at this point a procedure frequently employed in the algebraic approach. The procedure prescribes to consider these elements as given by their spectral expansions over projectors. The projectors belong on the one hand to $C^{*}$-algebra, and, on the other hand, they define the representation of these elements as linear operators in the Hilbert space. The elements admitting such a procedure are called adjoint to $C^{*}$-algebra. Thus, in this case, $\mathfrak{A}$ is the $C^{*}$-algebra supplemented with the adjoint elements.

It is convenient to pass on from the Hermitian elements $\hat{X}$ and $\hat{K}$ to the elements

$$
\hat{a}^{-}=\frac{1}{\sqrt{2 \omega}}(\omega \hat{X}+i \hat{K}), \quad \hat{a}^{+}=\frac{1}{\sqrt{2 \omega}}(\omega \hat{X}-i \hat{K})
$$

satisfying the commutation relation

$$
\left[\hat{a}^{-}, \hat{a}^{+}\right]=1
$$

and having simple time dependence

$$
\hat{a}^{-}(t)=\hat{a}^{-} \exp (-i \omega t), \quad \hat{a}^{+}(t)=\hat{a}^{+} \exp (+i \omega t) .
$$

Let us evaluate the generating functional of the Green's function. In the standard quantum mechanics, the $n$-time Green's function is defined by the formula

$$
G\left(t_{1}, \ldots t_{n}\right)=\left\langle 0\left|T\left(\hat{X}\left(t_{1}\right) \ldots \hat{X}\left(t_{n}\right)\right)\right| 0\right\rangle,
$$

where $T$ is the time ordering operator, and $|0\rangle$ is the quantum ground state.

According to Eqs. (17) and (21) the Green's function is defined in our approach with the formula

$$
\hat{p}_{0} T\left(\hat{X}\left(t_{1}\right) \ldots \hat{X}\left(t_{n}\right)\right) \hat{p}_{0}=G\left(t_{1}, \ldots t_{n}\right) \hat{p}_{0},
$$

where $\hat{p}_{0}$ is the spectral projector of $\hat{H}$ corresponding to the minimal value of energy.

It is easily seen that $\hat{p}_{0}$ is representable in the form

$$
\hat{p}_{0}=\lim _{r \rightarrow \infty} \exp \left(-r \hat{a}^{+} \hat{a}^{-}\right) .
$$

As mentioned above, the limit should be understood in the sense of the weak topology of $C^{*}$-algebra.

Let us first prove an auxiliary relation:

$$
\hat{E}=\lim _{r_{1}, r_{2} \rightarrow \infty} \exp \left(-r_{1} \hat{a}^{+} \hat{a}^{-}\right)\left(\hat{a}^{+}\right)^{k}\left(\hat{a}^{-}\right)^{l} \exp \left(-r_{2} \hat{a}^{+} \hat{a}^{-}\right)=0 \quad k, l \geq 0, \quad k+l>0 .
$$


Let $\Psi$ be a bounded positive linear functional. Then,

$$
\Psi(\hat{E})=\lim _{r_{1}, r_{2} \rightarrow \infty} \exp \left(-r_{1} k-r_{2} l\right) \Psi\left(\left(\hat{a}^{+}\right)^{k} \exp \left(-r_{1} \hat{a}^{+} \hat{a}^{-}\right) \exp \left(-r_{2} \hat{a}^{+} \hat{a}^{-}\right)\left(\hat{a}^{-}\right)^{l}\right) .
$$

Here, we used the continuity of the functional $\Psi$ and commutation relation (27). Next, taking into account $\left\|\exp \left(-r \hat{a}^{+} \hat{a}^{-}\right)\right\| \leq 1$, we obtain

$$
\begin{aligned}
|\Psi(\hat{E})| & \leq \lim _{r_{1}, r_{2} \rightarrow \infty} \exp \left(-r_{1} k-r_{2} l\right)\left|\Psi\left(\left(\hat{a}^{+}\right)^{k} \exp \left(-2 r_{1} \hat{a}^{+} \hat{a}^{-}\right)\left(\hat{a}^{-}\right)^{k}\right)\right|^{1 / 2} \\
& \times \quad\left|\Psi\left(\left(\hat{a}^{+}\right)^{l} \exp \left(-2 r_{2} \hat{a}^{+} \hat{a}^{-}\right)\left(\hat{a}^{-}\right)^{l}\right)\right|^{1 / 2} \\
& \leq \lim _{r_{1}, r_{2} \rightarrow \infty} \exp \left(-r_{1} k-r_{2} l\right)\left|\Psi\left(\left(\hat{a}^{+}\right)^{k}\left(\hat{a}^{-}\right)^{k}\right)\right|^{1 / 2}\left|\Psi\left(\left(\hat{a}^{+}\right)^{l}\left(\hat{a}^{-}\right)^{l}\right)\right|^{1 / 2}=0
\end{aligned}
$$

This proves equality (30).

Now we can check equality (29). In terms of the elements $\hat{a}^{+}, \hat{a}^{-}$, the Hamiltonian $\hat{H}$ is represented as $\hat{H}=\omega\left(\hat{a}^{+} \hat{a}^{-}+1 / 2\right)$. By (30)

$$
\lim _{r_{1}, r_{2} \rightarrow \infty} \exp \left(-r_{1} \hat{a}^{+} \hat{a}^{-}\right) \hat{H} \exp \left(-r_{2} \hat{a}^{+} \hat{a}^{-}\right)=\frac{\omega}{2} \lim _{r_{1}, r_{2} \rightarrow \infty} \exp \left(-\left(r_{1}+r_{2}\right) \hat{a}^{+} \hat{a}^{-}\right) .
$$

This proves equality (29).

Formula (28) implies that

$$
G\left(t_{1}, \ldots t_{n}\right) \hat{p}_{0}=\left.\left(\frac{1}{i}\right)^{n} \frac{\delta^{n}}{\delta j\left(t_{1}\right) \ldots \delta j\left(t_{n}\right)} \hat{p}_{0} T \exp \left(i \int_{-\infty}^{\infty} d t j(t) \hat{X}(t)\right) \hat{p}_{0}\right|_{j=0} .
$$

By Wick's theorem (see [31])

$$
\begin{gathered}
T \exp \left(i \int_{-\infty}^{\infty} d t j(t) \hat{X}(t)\right)= \\
=\exp \left(\frac{1}{2 i} \int_{-\infty}^{\infty} d t_{1} d t_{2} \frac{\delta}{\delta \hat{X}\left(t_{1}\right)} D^{c}\left(t_{1}-t_{2}\right) \frac{\delta}{\delta \hat{X}\left(t_{2}\right)}\right): \exp \left(i \int_{-\infty}^{\infty} d t j(t) \hat{X}(t)\right): .
\end{gathered}
$$

Here : : is the normal ordering operation, and

$$
D^{c}\left(t_{1}-t_{2}\right)=\frac{2}{\pi} \int d E \exp \left(-i\left(t_{1}-t_{2}\right) E\right) \frac{1}{\omega^{2}-E^{2}-i 0} .
$$

Making variations in $\hat{X}$ on the right-hand-side of (32) and taking into account (30), we obtain

$$
\begin{aligned}
\hat{p}_{0} T \exp \left(i \int_{-\infty}^{\infty} d t j(t) \hat{X}(t)\right) \hat{p}_{0} & =\exp \left(-\frac{1}{2 i} \int_{-\infty}^{\infty} d t_{1} d t_{2} j\left(t_{1}\right) D^{c}\left(t_{1}-t_{2}\right) j\left(t_{2}\right)\right) \\
\times \hat{p}_{0}: \exp \left(i \int_{-\infty}^{\infty} d t j(t) \hat{X}(t)\right): \hat{p}_{0} & =\hat{p}_{0} \exp \left(-\frac{1}{2 i} \int_{-\infty}^{\infty} d t_{1} d t_{2} j\left(t_{1}\right) D^{c}\left(t_{1}-t_{2}\right) j\left(t_{2}\right)\right) .
\end{aligned}
$$

Comparison with (31) gives

$$
G\left(t_{1} \ldots t_{n}\right)=\left.\left(\frac{1}{i}\right)^{n} \frac{\delta^{n} Z(j)}{\delta j\left(t_{1}\right) \ldots \delta j\left(t_{n}\right)}\right|_{j=0},
$$

where

$$
Z(j)=\exp \left(\frac{i}{2} \int_{-\infty}^{\infty} d t_{1} d t_{2} j\left(t_{1}\right) D^{c}\left(t_{1}-t_{2}\right) j\left(t_{2}\right)\right)
$$

is the generating functional.

As is known, consideration of field theory models within perturbation theory can be reduced to consideration of a multidimensional harmonic oscillator. For this reason, the above method for 
computing the generating functional of Green's functions can be directly generalized to quantum field theory models.

Our third example is the scattering of a quantum particle off two slits.

For simplicity, we consider two identical slits, $a$ and $b$. A homogeneous beam of particles is incident perpendicularly to the screen with the slits. We are interested in the interference pattern. Evidently, the structure of the pattern is determined by the probability distribution of the particle's momenta after the scattering.

Let $P\left(F_{k}\right)$ be the probability that a particle after scattering has momentum $K$. More accurately, that it has a momentum inside a small interval around $F_{k} . P\left(F_{k}\right)$ denotes the corresponding event in the sense of probability theory. Probability $F_{k}$ is conditional: before $F_{k}$ happens, the particle should come through one of the slits. This means that the coordinate of the particle at the moment of scattering should be either near $a$ (we denote this event $F_{a}$ ) or near $b$ (event $F_{b}$ ). The conditional probability is then denoted as $P\left(F_{k} /\left(F_{a}+F_{b}\right)\right)$.

By the probability theory (see, e.g., [25]):

$$
P\left(F_{k} /\left(F_{a}+F_{b}\right)\right)=\frac{P\left(F_{k} \cap\left(F_{a}+F_{b}\right)\right)}{P\left(F_{a}+F_{b}\right)} .
$$

$F_{k} \cap\left(F_{a}+F_{b}\right)$ means that two events have happened: $F_{k}$ and $\left(F_{a}+F_{b}\right)$. The symbol $\cap$ is used in order to take into account that the subset of elementary events corresponding to two simultaneous events coincides with the intersection of the subsets of elementary events corresponding to each of the events.

Since the events $F_{a}$ and $F_{b}$ do not intersect, we have

$$
P\left(F_{k} \cap\left(F_{a}+F_{b}\right)\right)=P\left(F_{k} \cap F_{a}+F_{k} \cap F_{b}\right)=P\left(F_{k} \cap F_{a}\right)+P\left(F_{k} \cap F_{b}\right) .
$$

On the other hand, because the beam is homogeneous,

$$
P\left(F_{a}+F_{b}\right)=P\left(F_{a}\right)+P\left(F_{b}\right)=2 P\left(F_{a}\right)=2 P\left(F_{b}\right) .
$$

With formulas (34) and (35), equality (33) can be transformed to

$$
P\left(F_{k} /\left(F_{a}+F_{b}\right)\right)=\frac{1}{2}\left[\frac{P\left(F_{k} \cap F_{a}\right)}{P\left(F_{a}\right)}+\frac{P\left(F_{k} \cap F_{b}\right)}{P\left(F_{b}\right)}\right] .
$$

The first term in the square bracket of the right-hand side comes from scattering off the slit $a$ and the second from scattering off the slit $b$. We did not obtain any interference. The mistake was that we used the formulas of probability theory without taking into account the specifics of applying probability theory to quantum events. Formula (33) is already erroneous. The problem is that the momentum and coordinate observables are incompatible. Therefore, as we explained in section 4 , no probability can be accorded to subset $F_{k} \cap\left(F_{a}+F_{b}\right)$.

A way to avoid this mistake is to take that the first stage of scattering consisting in the appearance of a particle near one of the slits is a preparation of a quantum state, which has its equivalence class of elementary states. Next, this class should be considered as the new space of elementary events. In this space, scattering at a given angle can be considered as an unconditional event.

We relate with event $F_{a}$ (particle appeared in domain near slit $a$ ) an observable $\hat{p}_{a}$ that can take two values, unity if particle passed through the slit, and zero otherwise. Evidently, this observable has the properties of a projector. Analogously, we introduce an observable $\hat{p}_{b}$. Clearly, the interference pattern is formed by particles whose elementary states correspond to the unit value of the observable $\hat{p}_{a}+\hat{p}_{b}$. We denote the set of these elementary state as $\Omega\left(p_{a}+p_{b}=1\right)$. This set corresponds to a quantum ensemble. Generally, such an ensemble is a mix of pure ensembles. The average values of observables over each of these pure ensembles are described by a linear 
positive functional. Thus, the average values of the observables over the quantum ensemble under consideration are also described by a linear positive functional.

Let $\Psi(\cdot)$ be such a functional corresponding to the set $\Omega\left(p_{a}+p_{b}=1\right)$. This functional satisfies the equality .

$$
\Psi\left(\hat{p}_{a}+\hat{p}_{b}\right)=\Psi(\hat{I})=1 .
$$

Thus, we can reproduce the derivation of formula (20) for this functional, and justify the equality

$$
\Psi(\hat{B})=\Psi\left(\left(\hat{p}_{a}+\hat{p}_{b}\right) \hat{B}\left(\hat{p}_{a}+\hat{p}_{b}\right)\right)
$$

for any observable $\hat{B}$. For the average value of the momentum, this formula yields

$$
\langle\hat{K}\rangle=\Psi\left(\hat{p}_{a} \hat{K} \hat{p}_{a}\right)+\Psi\left(\hat{p}_{b} \hat{K} \hat{p}_{b}\right)+\Psi\left(\hat{p}_{a} \hat{K} \hat{p}_{b}+\hat{p}_{b} \hat{K} \hat{p}_{a}\right) .
$$

The first two terms in the right-hand side are the contributions from the scattering off slits $a$ and $b$, respectively. The third term is the interference term.

\section{PROBLEM OF PHYSICAL REALITY}

Einstein, Podolsky, and Rosen 32 formulated the necessary principles for constructing a complete physical theory: (a) "every element of the physical reality must have a counterpart in the physical theory" and (b) "if, without in any way disturbing a system, we can predict with certainty (i.e., with probability equal to unity) the value of a physical quantity, then there exists an element of physical reality corresponding to this physical quantity."

Standard quantum mechanics does not comply with these theses. A single experiment has no copy in the mathematical formalism of standard quantum mechanics. Moreover, there is a strong opinion that such a copy cannot exist, and that even the objective physical reality that determines the outcome of a single physical experiment does not exist.

This opinion is backed with strong arguments. Probably, the best known one is based on the Bell inequality [33, 34]. Bell derived his inequality within the framework of the EPR theses. Following Bell, many versions of similar inequalities were suggested. Here, we consider the version suggested in [35. This version is usually denoted as CHSH.

Let a particle of zero spin decay into two particles $A$ and $B$, each having spin $1 / 2$. These particles move away from one another by large distances and are detected by devices $D_{a}$ and $D_{b}$, respectively. Device $D_{a}$ measures spin projection onto direction $a$ for particle $A, D_{b}$ measures spin projection onto direction $b$ for particle $B$. The corresponding observables are denoted as $\hat{A}_{a}$ and $\hat{B}_{b}$, and the outcomes of the measurements as $A_{a}$ and $B_{b}$.

Let us assume that the state of the initial particle is characterized with a physical reality, which is parameterized with parameter $\nu$. The same parameter is used to describe the physical realities that characterize the decay products. Correspondingly, the outcomes of measurements of observables $\hat{A}_{a}$ and $\hat{B}_{b}$ are functions of the parameter $\nu, A_{a}(\nu)$ and $B_{b}(\nu)$. Let the distribution of events in parameter $\nu$ be characterized with the probability measure $P(\nu)$ satisfying the standard conditions:

$$
\int P(d \nu)=1, \quad 0 \leq P(\nu) \leq 1 .
$$

Let us introduce the correlation function $E(a, b)$ :

$$
E(a, b)=\int P(d \nu) A_{a}(\nu) B_{b}(\nu)
$$

and consider the combination

$$
\begin{aligned}
N & =\left|E(a, b)-E\left(a, b^{\prime}\right)\right|+\left|E\left(a^{\prime}, b\right)+E\left(a^{\prime}, b^{\prime}\right)\right|= \\
& =\left|\int P(d \nu) A_{a}(\nu)\left[B_{b}(\nu)-B_{b^{\prime}}(\nu)\right]\right|+\left|\int P(d \nu) A_{a^{\prime}}(\nu)\left[B_{b}(\nu)+B_{b^{\prime}}(\nu)\right]\right| .
\end{aligned}
$$


For any directions $a$ and $b$,

$$
A_{a}(\nu)= \pm 1 / 2, \quad B_{b}(\nu)= \pm 1 / 2 .
$$

Thus,

$$
\begin{aligned}
N & \leq \int P(d \nu)\left[\left|A_{a}(\nu)\right|\left|B_{b}(\nu)-B_{b^{\prime}}(\nu)\right|+\left|A_{a^{\prime}}(\nu)\right|\left|B_{b}(\nu)+B_{b^{\prime}}(\nu)\right|\right]= \\
& =1 / 2 \int P(d \nu)\left[\left|B_{b}(\nu)-B_{b^{\prime}}(\nu)\right|+\left|B_{b}(\nu)+B_{b^{\prime}}(\nu)\right|\right] .
\end{aligned}
$$

Due to equalities (38), one of the two expressions

$$
\left|B_{b}(\nu)-B_{b^{\prime}}(\nu)\right|, \quad\left|B_{b}(\nu)+B_{b^{\prime}}(\nu)\right|
$$

vanishes, while another equals unity. We point out that both expressions involve the same value of $\nu$.

With these properties of expressions (40), inequality (39) implies the Bell inequality (CHSH):

$$
N \leq 1 / 2 \int P(d \nu)=1 / 2
$$

Within the standard quantum mechanics, the correlation function is easily calculated. The result is as follows:

$$
E(a, b)=-1 / 4 \cos \theta_{a b},
$$

where $\theta_{a b}$ is the angle between the directions $a$ and $b$. For the directions $a=0, b=\pi / 8, a^{\prime}=\pi / 4$, $b^{\prime}=3 \pi / 8$, we obtain

$$
N=1 / \sqrt{2},
$$

which is in contradiction with inequality (41).

Experiments agree with the quantum mechanical computations and disagree with the Bell inequality. Usually, these results are considered as a manifestation of the fact that no physical reality corresponding to a quantum mechanical system would predetermine the results of a measurement.

However, from the standpoint of modern probability theory, the above derivation of the Bell inequality is too naive. This derivation assumes that there is a probability distribution in the parameter $\nu$. By its meaning, this parameter marks an elementary event. As was pointed out (see Section 4), it is not always possible to assign a probability to an elementary event. The set of elementary events should be equipped with the structure of measurable space before one can speak about probability. In view of this, let us try to reproduce derivation of the Bell inequality using elementary state $\varphi$ in place of the parameter $\nu$.

According to the problem's conditions, the initial particle is in a particular quantum state. Thus, the space $\Omega\left(\varphi_{\eta}\right)$ of elementary events $\varphi$ is the equivalence $\{\varphi\}_{\varphi \eta}$. Thus, if an observable $\hat{A} \in \mathfrak{Q}_{\eta}$, then the observable will take one and the same value on all $\varphi \in\{\varphi\}_{\varphi \eta}$. The difference between elementary states $\varphi \in\{\varphi\}_{\varphi \eta}$ can be resolved by the values of observables $\hat{B} \notin \mathfrak{Q}_{\eta}$. It is easily seen that due to this difference the set $\varphi \in\{\varphi\}_{\varphi \eta}$ have the cardinality of continuum. Let us consider a subalgebra $\mathfrak{Q}_{\xi} \neq \mathfrak{Q}_{\eta}$ to justify this. Since the subalgebras $\mathfrak{Q}_{\xi}$ and $\mathfrak{Q}_{\eta}$ are maximal, there exists at least one observabable $\hat{B}$ such that $\hat{B} \in \mathfrak{Q}_{\xi}$ and $\hat{B} \notin \mathfrak{Q}_{\eta}$. The spectrum of this observable cannot be a single point. If $\lambda$ were this single point of the spectrum, then spectral radius of the element $\hat{B}-\lambda \hat{I}$ would vanish: $r(\hat{B}-\lambda \hat{I})=0$. However, $\|\hat{B}-\lambda \hat{I}\|=r(\hat{B}-\lambda \hat{I})$ for $C^{*}$-algebra. Thus, $\hat{B}=\lambda \hat{I} \in \mathfrak{Q}_{\eta}$. We conclude that there exist at least two elementary states $\varphi \in\{\varphi\}_{\varphi \eta}$ giving different values of observable $\hat{B}$. The same reasoning is applicable to any subalgebra $\mathfrak{Q}_{\xi} \neq \mathfrak{Q}_{\eta}$. Since the set of such subalgebras $\mathfrak{Q}_{\xi}$ has the cardinality of continuum, the set of different $\varphi \in\{\varphi\}_{\varphi \eta}$ will also have the cardinality of continuum.

Consider now formula (36)) for the correlation function. We need correlation functions for four combinations of the observables: $\hat{A}_{a} \hat{B}_{b}, \hat{A}_{a} \hat{B}_{b^{\prime}}, \hat{A}_{a^{\prime}} \hat{B}_{b}$, and $\hat{A}_{a^{\prime}} \hat{B}_{b^{\prime}}$. We are interested in the 
case when the directions $a, a^{\prime}, b$, and $b^{\prime}$ are not parallel to one another. In this case, the listed observables are not compatible with one another. Therefore, obtaining experimentally correlation functions requires four separate series of experiments. In reality, each of these series has a finite number of experiments. Ideally, they each would be a countable set of experiments.

We conclude that in experiment we deal not with a whole space $\Omega\left(\varphi_{\varphi \eta}\right)$ of elementary events, but with four random samples $\Omega_{a b}, \Omega_{a b^{\prime}}, \Omega_{a^{\prime} b}$, and $\Omega_{a^{\prime} b^{\prime}}$. Since these samples are countable even in the ideal case, and the set $\Omega\left(\varphi_{\varphi \eta}\right)$ has the cardinality of continuum, the probability that these samples contain common elements is zero. In addition, to make these samples measurable, one should select corresponding $\sigma$-algebras $\mathcal{F}_{a b}, \mathcal{F}_{a b^{\prime}}, \mathcal{F}_{a^{\prime} b}$, and $\mathcal{F}_{a^{\prime} b^{\prime}}$. These subalgebras differ from one another. Moreover, as discussed in section 4, they cannot be subalgebras of a single $\sigma$-algebra that would have a probability measure corresponding to it. In other words, each sample should have its own probability measure: $P_{a b}, P_{a^{\prime} b}, P_{a b^{\prime}}, P_{a^{\prime} b^{\prime}}$.

Now formula (36) ) takes the form

$$
E(a, b)=\int_{\Omega_{a b}} P_{a b}(d \varphi) \varphi\left(\hat{A}_{a} \hat{B}_{b}\right)
$$

and (37) becomes

$$
\begin{aligned}
N & =\left|\int_{\Omega_{a b}} P_{a b}(d \varphi) \varphi\left(\hat{A}_{a} \hat{B}_{b}\right)-\int_{\Omega_{a b^{\prime}}} P_{a b^{\prime}}(d \varphi) \varphi\left(\hat{A}_{a} \hat{B}_{b^{\prime}}\right)\right|+ \\
& +\left|\int_{\Omega_{a^{\prime} b}} P_{\hat{A}_{a^{\prime}} \hat{B}_{b}}(d \varphi) \varphi\left(\hat{A}_{a^{\prime}} \hat{B}_{b}\right)+\int_{\Omega_{a^{\prime} b^{\prime}}} P_{a^{\prime} b^{\prime}}(d \varphi) \varphi\left(\hat{A}_{a^{\prime}} \hat{B}_{b^{\prime}}\right)\right| .
\end{aligned}
$$

The same symbol $d \varphi$ is used in all four terms of formula (42). Despite this, the sets of elementary states corresponding to $d \varphi$ are different in different terms. They are elements of different $\sigma$ algebras. Moreover, the probability of sharing elements between these algebras vanishes. We conclude that, first, joining integrals under the modulus (42) is erroneous (this step was used in obtaining formula (37)). Second, forming pairs like the ones featured in formula (40) is erroneous. Thus, the proof of inequality (41) is invalid. Our conclusion is that if we relate physical reality to the elementary state, violation of Bell's inequality does not demonstrate in any way the contradictory nature of this notion.

Another argument against the applicability of the notion of physical reality to quantum physics is the Kochen-Specker no-go theorem [36]. The meaning of this theorem can be explained as follows. Consider as a physical system a particle with unit spin. Let $x, y, z$ be mutually orthogonal directions. Then observables $\hat{S}_{x}^{2}, \hat{S}_{y}^{2}, \hat{S}_{z}^{2}$ (spin projections squared) commute with each other. Thus, they are compatible and can be measured simultaneously. Assume that there exists a physical reality that predetermines unambiguously the outcome of a measurement for any direction. Measurement along some direction should yield zero, and along two others, unity. Select one of the latter directions and consider two directions (different from the initial directions) that are perpendicular to the selected direction. One of these directions should yield zero and another unity. Select the one yielding zero and repeat the whole procedure from the very beginning. It is possible to get in a finite number of such steps to a direction previously encountered. If this happens, it turns out that, if the first value corresponding to the direction was zero, the value obtained at the second encounter will be unity.

The conclusion drawn from this contradiction is that the physical reality governing the outcomes of measurements cannot exist. However, the above reasoning ignores completely the problem of measurability. Meanwhile, here we deal with two triples of directions: $x, y, z$, and $x, y^{\prime}, z^{\prime}$. Within each triple, all the directions are orthogonal, but there are nonorthogonal directions in different triples. Therefore, observables $\hat{S}_{x}^{2}, \hat{S}_{y}^{2}, \hat{S}_{z}^{2}$ and $\hat{S}_{x}^{2}, \hat{S}_{y^{\prime}}^{2}, \hat{S}_{z^{\prime}}^{2}$ belong to different commutative subalgebras of algebra $\mathfrak{A}$. Correspondingly, devices performing measurements that are compatible 
within each of the triples belong to different types. These devices should not necessarily yield the same outcome when measuring the observable $\hat{S}_{x}^{2}$. This was tacitly assumed in the above reasoning. Recall that the elementary state does not fix unambiguously the values of all observables. It fixes unambiguously the reading of all the devices of a specific type. For different types, these readings may differ. We conclude that within our approach the Kochen-Specker theorem does not exclude the existence of a physical reality related to the elementary state.

\section{PARADOXES}

Critics of standard quantum mechanics have pointed out a number of situations where quantum mechanical considerations lead to paradoxes. Here, we discuss only two such paradoxes, selecting the ones that are probably most frequently mentioned. The first is the Einstein-Podolsky-Rosen (EPR) paradox and the second, the Schrodinger cat paradox. It should be noted from the very beginning that the existence of paradoxes is denied by the most orthodox partisans of standard quantum mechanics. They claim that the correct use of the formulas of standard quantum mechanics does not lead to any paradoxes. So, before discussing specific paradoxes, we state our own position. It is as follows.

The formulas of standard quantum mechanics are certainly valid when applied to the description of quantum ensembles. They give correct values to the observable quantities and event probabilities also in the physical models considered by the authors of the paradoxes. Thus, only discussing individual events is of interest. Here, two positions are possible. On this, see review [37. First, it is possible to take that individual events are beyond the consideration of standard quantum mechanics. This position eliminates the subject of the discussion. However, individual events do exist. So, the question arises as to the completeness of the quantum mechanical description. Second, it is possible to take that quantum mechanics predicts only the probabilities of individual events, and the completeness of its description is exhausted by predictions of the probabilities. In this position, it is necessary to admit that probability is an independent entity of the individual event.

In modern probability theory, the latter is not the case. Recall that the notion of the space of elementary events comes before the notion of probability measure. Correspondingly, an individual event (elementary event) is considered as an element of a set (ensemble). And one and the same individual event can be considered as an element of different sets. Depending on this set, different probabilities (or no probability) may correspond to one and the same event.

The orthodox partisans of standard quantum mechanics reject this point of view and prefer to take that probability is a fundamental indefinable entity of any individual event, which is represented in the mathematical formalism of quantum mechanics with either a vector of a Hilbert space, or a density matrix. Formally, paradoxes are avoided in this way, but the physical essence of the phenomena remains beyond the framework of the discussion.

After these preliminary remarks, we begin the discussion of the paradoxes. We start with EPR. In the original paper [32, the paradox was considered on the example of measurements of coordinate and momentum. ohm suggested a simpler physical model [38]. The same problem is discussed on the example of measurements of spin projections onto different directions. Here, we discuss Bohm's version. In this case, we consider the physical system that was considered in the discussion of Bell's inequality.

Let a particle of spin 0 decay into two particles, $A$ and $B$, of spins $1 / 2$. After decay, the particles are separated by a large distance. According to standard quantum mechanics, the spin state of this system is described by the state vector

$$
|\Psi\rangle=\frac{1}{\sqrt{2}}\left[\left|A_{z}^{(+)}\right\rangle\left|B_{z}^{(-)}\right\rangle-\left|A_{z}^{(-)}\right\rangle\left|B_{z}^{(+)}\right\rangle\right]
$$


where $\left|A_{z}^{( \pm)}\right\rangle,\left|B_{z}^{( \pm)}\right\rangle$are the eigenvectors of the spin projection operators for axis $\mathrm{z}$ with the eigenvalues $+1 / 2$ and $-1 / 2$. This is a so-called entangled state. In this state, neither particle $A$ nor $B$ has any definite value of the spin projection onto axis $z$. The spin state of each particle can be described with a density matrix. For example, the density matrix for particle $A$ is

$$
\rho(A)=\frac{1}{2}\left[\left|A_{z}^{(+)}\right\rangle\left\langle A_{z}^{(+)}|+| A_{z}^{(-)}\right\rangle\left\langle A_{z}^{(-)}\right|\right] .
$$

This means that the particle has spin projections $+1 / 2$ and $-1 / 2$ with probabilities $1 / 2$.

Measure projection of spin onto axis $z$ for particle $B$ when two particles are in space-like domains. Let the result be $+1 / 2$. In this case, by the postulate of instant collapse of the quantum state (projection principle), the state $|\Psi\rangle$ will be instantly replaced with the state

$$
|\tilde{\Psi}\rangle=\hat{p}_{+}|\Psi\rangle / \sqrt{\left\langle\Psi\left|\hat{p}_{+}\right| \Psi\right\rangle}
$$

where $\hat{p}_{+}$is a projector of the form

$$
\hat{p}_{+}=\hat{I}_{A} \otimes\left|B_{z}^{(+)}\right\rangle\left\langle B_{z}^{(+)}\right|
$$

Here, $\hat{I}_{A}$ a is the unit operator in the space of states of particle $A$.

Substituting (45) into (44), we obtain $|\tilde{\Psi}\rangle=-\left|A_{z}^{(-)}\right\rangle\left\langle B_{z}^{(+)} \mid\right\rangle$. The density matrix of particle $A$ corresponding to this state is $\tilde{\rho}(A)=\left|A_{z}^{(-)}\right\rangle\left\langle A_{z}^{(-}\right|$. This means that any subsequent measurement of the axis $z$ spin projection for particle $A$ yields the value $-1 / 2$ with unit probability. This is what has been seen in the experiments. Thus, the projection principle is a good recipe. However, it would be nice to understand the physical mechanism that provides for the success of this recipe.

Two variations of such a mechanism are readily available. The first one is as follows. When created, particles acquire definite axis $z$ spin projections (of opposite sign). Before measuring this projection for particle $B$, we do not know the values of the projections. After the measurement for particle $B$, we know the projection for particle $A$ automatically. However, this mechanism contradicts standard quantum mechanics.

This is the case because quantum state $|\Psi\rangle$ can also be represented in the form

$$
|\Psi\rangle=\frac{1}{\sqrt{2}}\left[\left|A_{x}^{(+)}\right\rangle\left|B_{x}^{(-)}\right\rangle-\left|A_{x}^{(-)}\right\rangle\left|B_{x}^{(+)}\right\rangle\right]
$$

where the notations are the same as in (43), but projections onto axis $z$ are replaced with ones onto axis $x$. However, the observables corresponding to spin projections onto axes $z$ and $x$ are mutually incompatible, and, by the standard quantum mechanics, they cannot have definite values simultaneously.

The second version of the mechanism is as follows. After the decay, particles did not acquire any definite value of spin projection onto any axis. Due to the measurement of the projection onto a specific axis, they gained definite projections onto this axis. For particle $B$ that interacted with the measuring device, this mechanism is viable. However, how could such measurement influence particle $A$ located in a space-like domain with respect to the measuring device? This is not possible without a violation of the principles of relativity theory. Thus, both variants of the physical mechanism are unsound. This is the paradox.

Objecting against the paradox, Bohr pointed out [39] that it is not allowed in considering a correlated system to treat it as consisting of two separate independent parts. And any measurement acting on a part of the system should be considered as acting on the whole system. This reasoning is not very convincing in our opinion. Here, it is important that there are two types of correlations admitting rational explanations. The first type originates from interaction between the parts of the system. In the EPR case, such interaction would have to propagate faster than light. The second 
type originates from some constraint on the initial conditions for the particles under consideration. In the EPR case, such a constraint is in place since the particles originate from a decay of a single initial particle. However, the presence of the constraint is not enough for an unambiguous subsequent correlation of the particles. It is also necessary that the initial conditions would determine uniquely the subsequent time evolution of the particles. In this case, right after the creation, before measurement, particles $A$ and $B$ should posses a property that would determine unambiguously the result of the measurement. The latter contradicts the concept of standard quantum mechanics.

Surely, one could assume that there is a special quantum type of correlation that resists any rational treatment. Such an assumption would be the worst of all possible ones from the standpoint of science, since science strives to reduce the number of truths resisting rational understanding.

More apt are Fock's considerations [40]. Fock had taken that the notion of state does not have any objective meaning in the quantum case. Rather, it should be understood as a "data on the state." With this interpretation, the paradox can be avoided. However, it raises a question: "Is there anything objective about which we are collecting data?."

Within the approach of this paper, this "something" does exist. It is the elementary state. The elementary state is an objective characteristic of a physical system. It is independent of any knowledge of the system. In contrast, a quantum state, i.e., some equivalence class of elementary states is not a completely objective characteristic of a physical system. Instead, it is an objective characteristic of an ensemble of physical systems. A particular system of interest can be considered as an element of different ensembles (the freedom of choice). Correspondingly, the system will be characterized with different quantum states. For this reason, a quantum state involves a subjective factor.

Turning directly to the EPR paradox, we can give it the following interpretation. Before and after the decay of the original particle, the physical system has stable (zero) values of the observables $\hat{S}_{\mathbf{n}}$ (projections of the total spin onto the direction $\mathbf{n}$ ). After decay, the values of observables $\hat{A}_{\mathbf{n}}$ and $\hat{B}_{\mathbf{n}}$ (spin projections onto direction $\mathbf{n}$ for particles $A$ and $B$, respectively) satisfy the relation

$$
A_{\mathbf{n}}+B_{\mathbf{n}}=S_{\mathbf{n}}=0 .
$$

In principle, each of the observables $\hat{A}_{\mathbf{n}}$ and $\hat{B}_{\mathbf{n}}$ could be unstable. However, as pointed out in section 6 , for a two-level system (the case of a particle with spin $1 / 2$ ), these observables are stable. In the elementary state, incompatible observables can simultaneously have a definite value. However, these values cannot be measured simultaneously with any classical device. In a particular experiment, we can measure the observable $\hat{B}_{\mathbf{n}}$ for any but a single direction $\mathbf{n}$. This is the case because observables $\hat{B}_{\mathbf{n}}$ and $\hat{B}_{\mathbf{n}^{\prime}}$ are incompatible for different directions $\mathbf{n}$ and $\mathbf{n}^{\prime}$. Due to equality (46), in such a measurement we automatically measure $\hat{A}_{\mathbf{n}}$. This is the so-called indirect measurement. Thus, in this approach, the paradox is trivially solved.

Using this example, we can rationally treat the quantum state collapse phenomenon. Within the standard quantum mechanics, this phenomenon appears mystical.

Before measuring the spin of the $B$ particle, we know that our physical system is in the elementary state that belongs to the equivalence class characterized by the zero values of the observables $\hat{S}_{\mathbf{n}}$. In other words, we know that the system is in a singlet quantum state, but we do not know its elementary state. After measurement of observable $\hat{B}_{\mathbf{n}}$, due to equality (46), we gain knowledge not only on the value of this observable, but also on the value of the observable $\hat{A}_{\mathbf{n}}$. Therefore, after the measurement, we know that the system is in the elementary state that belongs to the equivalence class characterized by the values $A_{\mathbf{n}}=-B_{\mathbf{n}}\left(B_{\mathbf{n}}\right.$ known $)$ of the observables $\hat{A}_{\mathbf{n}}$ and $\hat{B}_{\mathbf{n}}$. Here, we assumed that measurement of $\hat{B}_{\mathbf{n}}$ is reproducible. Now we again do not know the elementary state of the system, but we know that the system is in a particular quantum state (of the type $|\tilde{\Psi}\rangle)$, formula (44)).

Due to interaction with the measuring device, the value of observables $\hat{B}_{\mathbf{n}^{\prime}}$ for directions $\mathbf{n}^{\prime} \neq \mathbf{n}$ changes uncontrollably. Because of this change, equality (46) breaks for such directions. This 
means that the system ceases to belong to the singlet state. In this way, all the features of the collapse of the quantum state are reproduced. We point out that before the measurement, we could describe the quantum state of particle $A$ with the density matrix

$$
\rho(A)=\frac{1}{2}\left[\left|A_{\mathbf{n}}^{(+)}\right\rangle\left\langle A_{\mathbf{n}}^{(+)}|+| A_{\mathbf{n}}^{(-)}\right\rangle\left\langle A_{\mathbf{n}}^{(-)}\right|\right]
$$

and after the measurement, with the density matrix

$$
\tilde{\rho}(A)=\left|-B_{\mathbf{n}}\right\rangle\left\langle-B_{\mathbf{n}}\right| .
$$

Despite the difference of the quantum states (47) and (48) before and after measurement (the former is mixed, the latter is pure), we cannot conclude that there is a change in the elementary state of particle $A$ due to the measurement. We simply gained extra information on this elementary state.

Equality (46) admits another useful interpretation. In the decay of the initial particle, each of the secondary particles measures the elementary state of its partner. This means that the elementary state of one of the particles is a negative copy of the elementary state of another particle. The creation of such a copy can be called a measurement with a quantum device. One particle is a quantum measuring device for another. In contrast to the measuring technique using a classical device, such measurement can fix unambiguously the elementary state of the particle under measurement. However, gaining access to the results of such a measurement requires a measurement in which the quantum device is studied with a classical device. Such a measurement results only in knowledge of the equivalence class containing the elementary state of the particle under measurement.

The scenario for the second paradox we will discuss was suggested by Schrodinger [41] (see also [42]). It goes as follows. A cat and a radioactive source of very small intensity are put in a box. When an atom decays in the source, the event is registered with a Geiger counter. The pulse from the counter comes through an amplifier to an automaton that breaks an ampoule with a poison. The cat is killed by the poison. The observer does not know if the decay had taken place or not. Thus, by the rules of quantum mechanics, the observer should describe the state of the complex system (the cat and the radioactive source) by a state vector that is a superposition of two quantum states: atom before decay and live cat plus decayed atom and dead cat. Superposition of the dead and alive cats looks very odd.

The opinion is sometimes expressed that the paradox disappears if one goes over from the description in terms of the vectors of a Hilbert space to description in terms of density matrix. Here, we should agree on the rules of our game. If we take that the density matrix describes an ensemble of physical systems, there will be no paradoxes. In this case, we deal not with a cat, but with an ensemble of cats, some of which are alive and some are dead. In this case, each cat is either live or dead, and which one we are dealing with is governed by probability theory. However, the meaning of the Schrodinger paradox is that we deal with a single cat. In this case, the above understanding of the density matrix is not appropriate. If we take that the density matrix describes the state of a single cat, a mixed state of a live and dead cat is not easier to contemplate than the superposition of such cats.

Certainly, there is no paradox if we take Fock's interpretation, i.e., if we take that the quantum mechanical state contains our knowledge of the objective state of a physical object. However, standard quantum mechanics does not take this attitude, and, in any case, it is not known if the objective state exists.

Within the framework of the elementary state concept, the paradox is trivially solved. The pair under study (the cat plus the radioactive atom) is in a specific elementary state. At a particular moment of time, the cat is either alive or dead in this state. There is no mixed elementary state of a live and dead cat. The quantum state describes an equivalence class of such elementary states. 
Among these elementary states, there are such that correspond to a cat alive at the given time, and there are such that correspond to the cat dead at the same moment of time.

When we place cat in the box, the information available to us pinpoints only the equivalence class, and not the elementary state. The equivalence class is fixed by the conditions we can classically register: at the moment the system was prepared, the cat was alive, and the atom had not decayed. On the other hand, the unambiguous evolution of the system is fixed namely by its elementary state. This state cannot be fixed unambiguously by classical measurements.

\section{FIELD-PARTICLE DUALITY}

In quantum case, elementary state $\varphi=\left[\varphi_{\xi}\right]$ of an individual physical system is a collection of functionals $\varphi_{\xi}(\cdot)$, each of which is a character of a maximal real commutative subalgebra $\mathfrak{Q}_{\xi}$ of algebra $\mathfrak{A}$. The set $\Xi(\xi \in \Xi)$ of these subalgebras has the cardinality of continuum. Thus, the elementary state is a field over the set $\Xi$ whose values are functionals.

Setting $\varphi$ is equivalent to setting $\varphi_{\xi}$ for every $\xi \in \Xi$. In turn, it suffices to set the value of $\varphi_{\xi}$ for every element of the generating set of the subalgebra $\mathfrak{Q}_{\xi}$ for setting $\varphi_{\xi}$. It is possible to take the view that a component of this functional-valued field corresponds to every generating set of the subalgebra $\mathfrak{Q}_{\xi}$. The value of functional $\varphi_{\xi}$ on a generating element can be considered as a value of the component of the field $\varphi$ at the point $\xi$. Thus, $\varphi$ is a real $c$-number multicomponent field over the set $\Xi$.

Correspondingly, even a quantum system that is conventionally considered as a system of finite number of degrees of freedom (e.g., harmonic oscillator), is, in fact, a field system, i.e., a system with an infinite number of degrees of freedom. This implies that the elementary state of any quantum system may principally encode an infinite amount of information. However, it is not possible to really use this infinite volume of information. This is the case because we should have the possibility to control the information with classical devices to make it usable. However, classical devices are unable to tell the difference between different elementary states; they can tell the difference only between the equivalence classes corresponding to the quantum states. Thus, the volume of controllable information turns out to be finite. Still, it can be much larger than for classical physical systems. This is the physical prerequisite for the possibility of constructing quantum computers.

The elementary state of any physical system is also a field over Minkowski space. This is the case because the systems that are traditionally considered in quantum mechanics as pointlike are in fact distributed over Minkowski space.

For example, consider an electron that scatters "elastically" off a nucleus. In reality, such scattering is accompanied by bremsstrahlung of soft photons. However, the energy and other observables of these photons are beyond the sensitivity of the measuring devices. In other words, electron scattering is accompanied by the emission of soft photons. Although this field is not measurable with classical devices, it plays an important role in theory. Without it the theory suffers from infrared divergences. At one time, this situation was called the infrared catastrophe.

The infrared catastrophe can be avoided only if the number of soft radiated photons is infinite. This means that the electron is accompanied by an effective classical field. Clearly, any other process involving the creation of virtual electron-positron pairs will be accompanied by such a classical electromagnetic field (effective). A similar situation takes place in quantum chromodynamics. In chromodynamics, the major hopes for explaining quark confinement are related precisely to soft partons.

We conclude that elementary state $\varphi$ of any quantum system is described with a $c$-number field over the set $\Xi$ and Minkowski space. This field has all the attributes of a real classical field. If we assume the classical paradigm, we should take that there exists a material field mathematically 
accounted for by $\varphi$. This effective classical field can include the classical component of the electromagnetic field, the gravitation field, or some other field. Concrete physical realization of this field is not important for us. We will call it the phase field, by analogy with the phase space that determines state of the system. We point out that the field is classical and, therefore, the quantum relation between energy and frequency may not hold.

Assumption of the material existence of a phase field may help to solve one of the problems of quantum theory, the problem of wave-particle duality. Here, we call this notion field-particle duality. The field properties of a quantum system are naturally related to the phase field, i.e., to the elementary state.

The particle properties of a quantum system mean the following. The physical system has local observables, i.e., observables associated with a bounded domain of Minkowski space. These observables or, more accurately, their complex combinations form the algebra of local observables. Note that the algebra of local observables is one of the major notions of the traditional algebraic approach to quantum field theory (see [43, 44, 6, 7]). There exist stable (meaning frequently encountered) sets of values of local observables, which we treat as quantum particles of particular species: electrons, photons, nuclei, atoms, etc.

Measuring devices perceive these observables as an indivisible whole. In this, the particle properties of quantum systems are exhibited. The reaction of a measuring device is determined by the elementary state of the system (by the phase field). In turn, the structure of (the value taken by) the phase field is determined by the spectra of the corresponding observables. We point out that a point of the spectrum is an indivisible whole. In this way, the particle and field properties turn out to be tightly intertwined in a quantum system.

In standard quantum mechanics, the quantum state of a physical system is also associated with a $c$-number field, with the wave function. However, the wave function takes values in complex numbers. So, it cannot correspond directly to a material field. In our approach, the wave function is related only to probability, and the relation is rather indirect. Namely, the average values of the observables may be represented in the form of expectation values of linear operators in a Hilbert space (the averages by their nature are not such objects). In turn, the vectors of Hilbert space may be represented as wave functions.

The phase field may be considered as a material substance bearing information on the physical state of a quantum object. It should be coordinated (coherent) with the associated quantum object. If this condition is fulfilled, it is possible to construct a plausible model of the measurement process based on the phase field. Recall that the lack of such a model is usually put forward as the reason behind all the hitches that are present in standard quantum mechanics.

Let us describe such a model. (See also on this subject the papers of Blokhintsev [20, 21, 22, 23]].) The measuring device consists of an analyzer and detector. Sometimes these components are combined. The analyzer is a device with a single input channel and a number of output channels. If a device is dedicated to measurement of an observable $\hat{A}$, each output channel corresponds to a particular region of the spectrum of the observable, i.e., each output channel corresponds to an equivalence class of elementary states.

The phase field associated with the object under measurement excites collective oscillations in the device that are coherent with the field. The oscillations may be very weak, but due to the coherence they interact with the quantum object in a resonance way. Microscopic description of such interaction is practically inaccessible. However, the result of this interaction can be described with a boundary condition. If the quantum state of the object under measurement descries the equivalence class corresponding to an output channel, the object unavoidably ends up in this output channel. If the object under measurement is in a quantum state that does not correspond to any of the output channels, the analyzer turns out to be a bifurcation domain for the object. In this case, the resonance interaction of the object with the oscillations of the analyzer excited by the phase field is the random force directing the object into a particular output channel, namely, into 
the channel corresponding to the equivalence class that includes the elementary state of the object under measurement.

Here, the localization domain of the quantum object is the domain of localization of its local observables that can be registered by classical measuring devices. In the following, this localization domain is called the kern of the quantum object. At the same time, as mentioned earlier, any quantum object is accompanied by a field that, on the one hand, is not registered by the measuring devices, and, on the other hand, is a component of the phase field. Therefore, an analyzer may become the bifurcation domain for the phase field.

An analyzer is a classical object. Interaction of the phase field with a classical object may be of two types. Under the interaction of the first type, the coherence of the phase field with the emitting object remains intact; under the second, interaction breaks the coherence. Since we assume that the phase field excites oscillations in the analyzer that are coherent with the field, we are to take that interaction with the analyzer does not break the coherence of the field. We also take that the impact of the quantum object on the analyzer is not registered macroscopically. The registration tales place in the detector. The detector is a classical system that is put into a state of unstable equilibrium. The detector interacts with the kern of the quantum object. The interaction results in bringing the detector out of equilibrium. A catastrophic, macroscopically detectable process develops in the detector. The detector (detectors) is placed at one (several) output channels of the analyzer. Engaging of detector results in fixation of the output channel of the analyzer that the kern of the quantum object has hit.

In this way, the value of the observable $\hat{A}$ of the quantum object becomes fixed. At the same time, an equivalence class that includes the elementary state of the object under measurement becomes fixed.

The reverse impact of the detector onto the quantum object is also strong. For an irreproducible measurement, the elementary state of the object is changed completely. For a reproducible measurement, coherence is broken anyway, but the elementary state of the quantum object remains in the equivalence class corresponding to the output channel that passed through itself the kern of the quantum object. The kern of the quantum object and other components of the phase field moving along with the kern and passed through other output channels of the analyzer cease to be coherent parts of phase field.

If a detector is placed on an output channel that did not convey the kernel through, the detector experiences only a weak impact from the part of the phase field that passed though the channel. The catastrophic process does not develop in the detector, and no macroscopic effect is registered. Despite this, feedback from the detector onto the phase field is substantial. The field in this channel loses coherence with the kernel of the quantum object, and with the parts of the phase field that have passed through other channels.

If detectors are not mounted in any of the output channels, it is in principle possible to unite back all the parts of the phase field transmitted through different channels. They will coherently add up, and the initial elementary state may be reconstructed. If there is a detector in one of the channels, the corresponding part of the phase field cannot take part in the coherent addition. Effectively, from the standpoint of elementary state of the quantum object, this part of the phase field is lost.

Thus, a part of the phase field that determines the elementary state of the quantum object may effectively "disappear" in two cases. Either a change of the state (with decoherence) of the disappeared part of the field takes place, or the disappeared part does not change, but the state of the kernel of the quantum object changes. In both cases, there is a change in the structure of the phase field, which is coherent with the kernel. Namely, such a field determines the elementary state of the quantum object. Under a change of elementary state, a change of quantum state naturally occurs. This change has all the features of the collapse of quantum state induced by measurement. A similar model of measurements is given in review [45]. 
The phase field performs the functions usually ascribed to hidden parameters. However, in contrast to the situation with hidden parameters, we point out the way to construct a mathematical image of this field. For this reason, mathematically, there is no existence problem for this field. All the objections that are usually put forward against hidden parameters are not valid in the case of a phase field. However, surely, the existence of material realization of the phase field remains a hypothesis at the moment.

We point out that the status of this hypothesis differs from the one of the statements from the above postulates. The latter abstract the results obtained in physical observations. Based on these postulates, the mathematical formalism of our scheme is constructed. For this construction, the hypothesis we discuss is not needed. On the contrary, within the classical paradigm, the hypothesis can be derived from the mathematical formalism constructed. More accurately, the phase field seems to be a most viable candidate for the material realization of the mathematical notion of elementary state. At the same time, the most viable does not mean the only possible.

In contrast to hidden parameters, the phase field is partially observable. It influences the kernel behavior of a quantum object, if the kernel is coherent with the phase field. In turn, detector of the classical measuring device is sensitive to the kernel. A classical device is not sensitive in any way to a phase field that lost coherence with its kernel. However, this does not mean that this field has disappeared. It may manifest itself as dark matter.

The concept that any quantum object consists of a kernel and a phase field allows for very intuitive interpretation of the so-called delayed-choice experiment. The idea of this experiment was suggested by Wheeler [46]; the experiment was performed independently by two collaborations [47, 48 .

The scheme of the experiment is as follows. Figs. l(a) and l(b) show two experimental setups. $M_{1}$ and $M_{4}$ are two semireflective mirrors, $M_{2}$ and $M_{3}$ are normal mirrors, $D_{A}$ and $D_{B}$ are detectors.

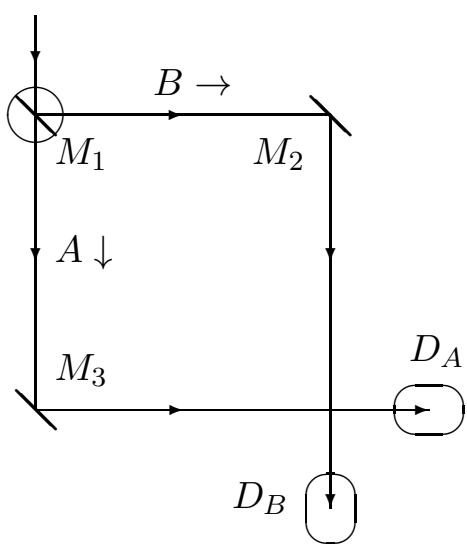

(a)

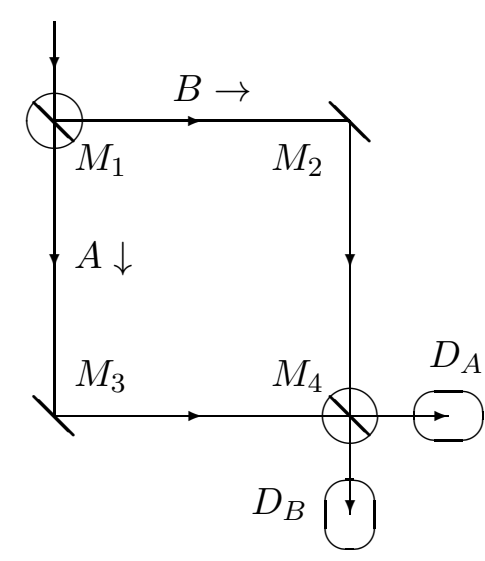

(b)

Fig. 1. Delayed-choice experiment.

A pulse of light arrives onto semireflective mirror $M_{1}$. The pulse splits into two parts. One of the parts traverses path $A$, another, path $B$. In the case of Fig. la, these parts do not mix with one another and are registered independently by detectors $D_{A}$ and $D_{B}$. In the case of Fig. lb, both parts get to the semireflective mirror $M_{4}$, where interference between the two parts takes place. Since the phase of oscillations changes by $\pi / 2$ at reflection, and does not change in passing through a semireflective mirror $M_{4}$, all of the light pulse arrives at the detector $D_{B}$ after the interference. 
Wheeler's idea consists in making the pulse weak and short, and the intervals between pulses large enough. In this way, not more than a single photon is localized in the detector at any moment. Second, the semireflective mirror $M_{4}$ is removable. By the choice of the experimenter, it is either set or removed in the time span between the moments when the photon passes the semireflective mirror $M_{1}$ and reaches the domain of the semireflective mirror $M_{4}$.

If the semireflective mirror $M_{4}$ is not put in (Fig. la), each of the detectors $D_{A}$ and $D_{B}$ are engaged with probability $1 / 2$ for each pulse. This case is easy to interpret if we consider the photon as a particle. Then it takes either path $A$ or path $B$ after passing semireflective mirror $M_{1}$ with probabilities $1 / 2$. Correspondingly, it hits either detector $D_{A}$ or detector $D_{B}$.

If the semireflective mirror $M_{4}$ is put in (Fig. lb), only the detector $D_{B}$ responds. This case is easy to interpret if the photon is considered as a wave. Then the wave is split by semireflective mirror $M_{1}$ into two components propagating via the paths $A$ and $B$. The components interfere at the semireflective mirror $M_{4}$. After the interference, the wave propagates after semireflective mirror $M_{4}$ only to the detector $D_{B}$.

The results of the experiments confirmed Wheeler's expectations completely. This means that the photon behaves as a particle or as a wave, depending on the situation. The zest of Wheelers idea is that when a photon interacts with the semireflective mirror $M_{1}$; it cannot know how it should behave-as a particle or as a wave. To decide on this, it should foresee the whim of the experimentalist.

An orthodox partisan of standard quantum mechanics will say right away that the above intuitive interpretations are wrong, because they are classical. In reality, a photon is not a particle and not a wave. After passing semireflective mirror $M_{1}$, neither a photon nor its components propagate either along path $A$ or along path $B$. What takes place in semireflective mirror $M_{1}$ is really the spitting of the wave function of the initial photon into parts corresponding to the paths $A$ and $B$. Next, in semireflective mirror $M_{4}$, if it is present, the coherent addition of these parts of the wave function takes place. Such addition yields a correct account of the experiment.

Such an explanation is acceptable if one takes that wave function describes a physical field. However, standard quantum mechanics rejects this assumption. And it does so very justly, because, if nothing else, the wave function takes values in complex numbers. The wave function is probability amplitude. It is a purely mathematical construct. As such, it cannot interact either with semireflective mirror $M_{1}$ or with semireflective mirror $M_{4}$. The evolution of the wave function should describe a physical process. Standard quantum mechanics is unable to explain the nature of this process.

The above experiment seems to confirm one of the major precepts of quantum mechanics: a quantum object cannot have any definite trajectory. Despite this precept, almost all experiments in particle physics (which are surely quantum objects) are based on analysis of these non-existing trajectories. The situation is paradoxical: theoreticians and experimenters in elementary particle physics play simultaneously the same game obeying different rules.

The hypothesis of the phase field supports the experimenter's interpretation. After the semireflective mirror $M_{1}$; the photon's kernel propagates either by path $A$ or by path $B$ depending on its elementary state. The phase field splits into two components on semireflective mirror $M_{1}$ the components propagate by different paths. In the mirror $M_{4}$, if it is present, these two components of the phase field add up coherently into the resulting phase field (elementary state) that directs the photon's kernel to the detector $D_{B}$.

Also, within our hypothesis, the scattering of a quantum object off two slits can be interpreted. In the experiment, a distinct interference pattern is observed. The interference pattern is observed even in the case when the intensity of the particle beam is so weak that not more than one particle is located in the experimental setup [49]. In this case, it is not possible to explain the interference by interaction between incident particles. Rejecting verbal embellishments, the standard interpretation of this experiment reduces to the following. Indivisible before the slits, the quantum object passes 
simultaneously through both slits, which are separated by a macroscopic distance; after that, the object stays indivisible.

Our interpretation is much more intuitive. The phase field of the quantum object excites weak collective oscillations in the screen. These oscillations generate a secondary classical field. Oscillations in various parts of the screen are coherent with one another. Because of this, the components of the secondary field radiated by different parts of the screen add up coherently. The kernel of the quantum object approaches as a whole the slits. The kernel may scatter off the slit at different angles. From the standpoint of standard quantum mechanics, this process has no definite cause. In terms of this paper, the slit is a bifurcation domain for the quantum object. The behavior of a particular quantum object in such a domain is governed by a random force. This random force results from interaction between the kernel and the secondary classical field radiated by the screen. This field is very weak, but it is coherent with the kernel. Because of this, its interaction with the kernel is a resonance one. The structure of the resonance field at the location of the kernel changes when one of the slits is closed. In view of this, the ensemble of quantum objects scattered off two slits is not a simple mix of ensembles of quantum objects scattered separately by each of the slits. As a result, the interference pattern appears.

Surely, these qualitative considerations are insufficient for quantitative computation of the interference pattern. For such a computation, mathematical formalism of the standard quantum mechanics can be used. This is the case because the interference pattern appears only if a very large number of quantum objects is scattered (see [49]). In this case, we deal not with a single elementary state, but with a large number of elementary states. By the law of large numbers, this collection of elementary states can be replaced with an equivalence class, i.e., with a quantum state. Here, we get to the domain of applicability of the standard quantum mechanics.

\section{CONCLUSIONS}

The approach we have presented by no means rejects standard quantum mechanics. The original researches did a splendid job in developing quantum mechanics, but they started the construction from the first floor-from the description of probabilities and average values. Because of this, the stability of the construction required the introduction of a number of props in the form of "principles": the superposition principle, the uncertainty principle, the complementarity principle, the projection principle, the indistinguishability principle, and the no trajectories principle. All of these are far from obvious, and appear to be rather artificial. On the other hand, a number of principles-causality, formal logic-had to be rejected, despite the fact that they had stood the test of all of the previous history of mankind. It may seem that these two are only deeply rooted misconceptions. Examples of such misconceptions are available. On the other hand, we should do our best to keep them alive. The scheme we advocate is an attempt in this direction.

In a way, the relation of our scheme to the standard quantum mechanics resembles the one of statistical physics to thermodynamics. Thermodynamics can be constructed on its own, without reference to statistical physics. Historically, this indeed took place. It is also true, however, that thermodynamics gained extra momentum after its basic principles had lost the status of fundamental laws of nature. Instead, these principles became derivable from the more fundamental laws of statistical physics. In this case, physics gained the possibility to use to the full extent the powerful formalism of probability theory.

A distinctive feature of statistical physics is that it involves the notion of elementary event. In statistical physics, which studies physical systems consisting of a huge number of components, an elementary event is the state of all the components. In practice, one cannot observe or fix it. However, its existence is a matter of principle, because it allows one to use probability theory.

In standard quantum mechanics, as it was in thermodynamics, the notion of elementary event is absent. The principal feature of our approach is that it introduces the notion of elementary state, 
which can be considered as an elementary event. As in statistical physics, an elementary state is unobservable, but allows one to use to the full extent the classical formal logic and the standard probability theory.

Using the notion of elementary state, it is possible to establish the limits of applicability of the mathematical formalism of standard quantum mechanics. Within these limits is the study of the ensembles of quantum objects that can be described by a quantum state. It is a very important type of ensembles, but it is far from being the most general type. In particular, standard quantum mechanics is unable to describe individual quantum objects. At the same time, the formalism of modern probability theory is powerful enough to describe the behavior of ensembles of a more general type. Thus, we hope that the range of applicability of quantum mechanics may be extended.

Moreover, with an elementary state that corresponds to individual quantum object, it is possible to give an intuitive interpretation of quantum phenomena. The notion of elementary state allows one to get rid of so-called quantum logic and quantum probability theory. Both schemes are presently more advertisements than developed schemes. Intuitively, we perceive them as unfeasible.

The scheme we have suggested makes it possible to give a unified treatment of quantum and classical systems. Due to this, a question rather painful for the standard quantum mechanics-which mechanics, quantum or classical, is logically primary-is resolved.

Finally, our scheme does not reject the causality principle, treating it as a principle shared by both quantum and classical physics.

\section{References}

[1] J. von Neumann, Mathematical Foundations of Quantum Mechanics (Princeton Univ. Press, Princeton, 1955; Nauka, Moscow, 1964).

[2] I. Segal, Mathematical Problems of Relativistic Physics (Amer. Math. Soc, Providence, 1967; Mir, Moscow, 1968).

[3] L. D. Landau and E. M. Lifshitz, Course of Theoretical Physics, Vol. 3: Quantum Mechanics: Non-Relativistic Theory, 3rd ed. (Nauka, Moscow, 1974; Pergamon, New York, 1977).

[4] A. N. Kolmogorov, Foundations of the Theory of Probability, 2nd ed. (2nd ed., Nauka, Moscow, 1974; Chelsea, New York, 1956).

[5] A. S. Holevo, Probabilistic and Statistical Aspects of Quantum Theory (Nauka, Moscow, 1980; North-Holland, Amsterdam, 1982).

[6] G. Emch, Algebraic Methods in Statistical Mechanics and Quantum Field Theory (Wiley, New York, 1972; Mir, Moscow, 1976).

[7] . S. S. Horuzhy, Introduction to Algebraic Quantum Field Theory (Kluwer, Dordrecht, 1988; Nauka, Moscow, 1986).

[8] N. N. Bogoliubov et al., General Principles of Quantum Field Theory (Nauka, Moscow, 1987; Kluwer, Dordrecht, 1990).

[9] D. A. Slavnov, Teor. Mat. Fiz. 129 (1), 87 (2001) [Theor. Math. Phys. 129, 1385 (2001)].

[10] D. A. Slavnov, Teor. Mat. Fiz. 132 (3), 434 (2002) [Theor. Math. Phys. 132, 1264 (2002)].

[11] D. A. Slavnov, Teor. Mat. Fiz. 142 (3), 510 (2005) [Theor. Math. Phys. 143, 431 (2005)].

[12] U. Rudin, Functional Analysis (McGraw-Hill, New York, 1973; Mir, Moscow, 1975).

[13] M. A. Najmark, Normed Algebras 3rd ed. (Nauka, Moscow, 1968; Wolters-Noordhoff, Groningen, 1972).

[14] O. Bratteli and D. Robinson, Operator Algebras and Quantum-Statistical Mechanics (Springer, New York, 1979; Mir, Moscow, 1982).

[15] J. S. Dixmier, Les $C^{*}$ algebres et leurs representations (Gauthier-Villars, Paris, 1969). 
[16] N. Bohr, "Quantum Postulate and Recent Development of Atomic Theory," in Atomic Theory and Description of Nature (Cambridge Univ. Press, Cambridge, 1934), p. 52.

[17] A. Zeilinger, Rev. Modern. Phys. 71, S289 (1999).

[18] V. A. Fock, Bases of Quantum Mechanics (Nauka, Moscow, 1976) [in Russian].

[19] N. Bohr, "Discussion with Einstein on Epistemological Problems in Atomic Physics," in Albert Einstein: Philosopher-Scientist, Ed. by PA. Schlipp (La Salle, Open Court, 1949), p. 201.

[20] D. I. Blokhintsev, Bases of Quantum Mechanics (Nauka, Moscow, 1976) [in Russian].

[21] D. I. Blokhintsev, Usp. Fiz. Nauk 122 (4), 745 (1977) [Phys.-Usp. 20, 683 (1977)].

[22] D. I. Blokhintsev, Principal Questions of Quantum Mechanics (Nauka, Moscow, 1987) [in Russian].

[23] D. I. Blokhintsev, Quantum Mechanics. Lectures about Selected Questions (Mosk. Gos. Univ., Moscow, 1988) [in Russian].

[24] D. A. Slavnov, Teor. Mat. Fiz. 136 (3), 437 (2003) [Theor. Math. Phys. 136, 1237 (2003)].

[25] J. Neveu, Bases mathematique du calcul des probilites (Masson, Paris, 1968; Mir, Moscow, 1969).

[26] Yu. V. Prokhorov and Yu. A. Rozanov, Probability Theory (Nauka, Moscow, 1967; Springer, Berlin, 1969).

[27] P. Jordan, Z. Phys. 37, 285 (1933).

[28] D. Bohm, Phys. Rev. 85, 166 (1952).

[29] R. F. Streater and A. S. Wightman, PCT, Spin and Statistics and All That (Benjamin, New York, 1964; Nauka, Moscow, 1966).

[30] M. Born, Z. Phys. 37, 863 (1926); 38, 803 (1926); 40, 167 (1927).

[31] N. N. Bogoliubov and N. N. Bogoliubov, Jr., Introduction to Quantum Statistical Mechanics (Nauka, Moscow, 1984; World Scientific, Singapore, 1982).

[32] A. Einstein, B. Podolsky, and N. Rozen, Phys. Rev. 47, 777 (1935).

[33] J. S. Bell, Physics 1, 195 (1965).

[34] J. S. Bell, "On the Einstein-Podolsky-Rosen Paradox," in Speakable and Unspeakable in Quantum Mechanics: Collected Paper on Quantum Philosophy (University Press, Cambridge, 1993), p. 139.

[35] J. F. Clauser, M. A. Horne, A. Shimony, and R. A. Holt, Phys. Rev. Lett. 23, 880 (1969).

[36] S. Kochen and E. P. Specker, J. of Math. Mechanics 17, 59 (1967).

[37] D. Home and M. A. B. Whitaker, Phys. Rep. 210, 223 (1992).

[38] D. Bohm, Quantum Theory (Prentice-Hall, Englewood Cliffs, N.Y., 1951; Nauka, Moscow, 1965).

[39] N. Bohr, Phys. Rev. 48, 696 (1935).

[40] V. A. Fock, Usp. Fiz. Nauk 16 (4), 436 (1936).

[41] E. Schrodinger, Naturwissenschaften 23, 807 (1935).

[42] V. V. Belokurov, O. D. Timofeevskaya, and O. A. Khrustalev, Quantum Teleportation Usual Wonder (Regulyarnaya i khaoticheskaya dinamika, Izhevsk, 2000) [in Russian].

[43] H. Araki, Progr. Theor. Phys. 32 (5), 844 (1964).

[44] R. Haag and D. Kastler, J. Math. Phys. 5 (7), 848 (1964).

[45] M. Namiki and S. Pascazio, Phys. Rep. 232, 301 (1993).

[46] A. Wheeler, Mathematical Foundation of Quantum Theory (Academic, New York, 1978), p. 9.

[47] .. Alley, . G. Jakubowicz, and W. Wicks, in Proc. of Second Intern. Symp. on the Foundations of Quantum Mechanics (Physical Society of Japan, Tokyo, 1987), p. 36.

[48] T. Hellmuth et al., Phys. Rev. A 35, 2532 (1987).

[49] A. Tonomura, Phys. Today 41, 22 (1990). 